\title{
LA-UR-17-24503
}

Approved for public release; distribution is unlimited.

Title: $\quad$ Uncertainty in the use of MAMA software to measure particle morphological parameters from SEM images

Author(s): $\quad$ Schwartz, Daniel S.

Tandon, Lav

Intended for: Report

Issued: 
Disclaimer:

Los Alamos National Laboratory, an affirmative action/equal opportunity employer, is operated by the Los Alamos National Security, LLC for the National Nuclear Security Administration of the U.S. Department of Energy under contract DE-AC52-06NA25396. By approving this article, the publisher recognizes that the U.S. Government retains nonexclusive, royalty-free license to publish or reproduce the published form of this contribution, or to allow others to do so, for U.S. Government purposes. Los Alamos National Laboratory requests that the publisher identify this article as work performed under the auspices of the U.S. Department of Energy. Los Alamos National Laboratory strongly supports academic freedom and a researcher's right to publish; as an institution, however, the Laboratory does not endorse the viewpoint of a publication or guarantee its technical correctness. 


\section{Uncertainty in the use of MAMA software to measure particle morphological parameters from SEM images}

\section{Part 1. Uncertainty for Level of Rigor 1}

Daniel S. Schwartz and Lav Tandon, Los Alamos National Laboratory

\section{Background}

The MAMA software package developed at LANL is designed to make morphological measurements on a wide variety of digital images of objects. At LANL, we have focused on using MAMA to measure scanning electron microscope (SEM) images of particles, as this is a critical part of our forensic analysis of interdicted radiologic materials. In order to successfully use MAMA to make such measurements, we must understand the level of uncertainty involved in the process, so that we can rigorously support our quantitative conclusions.

The process of using MAMA to analyze particles with SEM can be divided into three distinct steps:

1. Sampling a subset of the particles to be analyzed and fixing them appropriately for observation by microscopic means. Fixation involves loading particles (typically 10,000 to 100,000 ) onto an adhesive substrate, and may include coating the mounted particles with a thin conductive layer of gold or carbon.

2. Creating images of the particle subset.

3. Making morphological measurements on the images, which includes qualitative observations of particle shape and quantitative, statistical measurements of desired morphological parameters.

Each of these steps introduces uncertainty into the morphological quantifications. As all the steps involve a large number of sub steps, we decided to quantify the uncertainty using a round-robin approach rather than attempting to quantify the uncertainty of each step. Expert analysts from 4 different laboratories were given particle measurement tasks, described in detail in the next section. Uncertainty was determined as the maximum variation observed between measurements for each morphological parameter, at three well-defined levels of rigor. This is described in detail in the next section.

\section{Phase 3 Task 2 Exchange Program}

A group of expert particle analysts from LANL and 3 other national laboratories agreed to participate in a round robin exchange of images and materials. The round robin participants included:

1. LLNL (R. Kips, point of contact) 
2. PNNL (E. Buck, point of contact)

3. SRNL (J. Venzie, point of contact)

Each of these laboratories and points of contact has extensive experience with particle analysis and forensics of radiological materials using SEM, as well as expertise in the use of the MAMA software.

NIST standard reference material SRM 1984 [Thermal Spray Powder - Particle Size Distribution, Tungsten Carbide/Cobalt (Acicular)] was chosen as an exchange material for this effort. SRM 1984 is a suitable powder set, as it bears some resemblance to the types of particles that could be involved in actual radiological particle forensics in both shape and size, but is non-radioactive, relatively inert, and safe to handle. Several 14 g bottles of SRM 1984 were procured from NIST and one bottle was distributed to each of the participating laboratories.

The exchange program consisted of data and material exchanges at three levels of rigor, designed to determine uncertainties associated with different parts of the particle analysis process.

1. Level of Rigor 1. Each laboratory was given a set of 10 SEM images (Figure 1) of SRM 1984 made at LANL, with instructions to use MAMA version 2.0.2 to measure $>400$ particles, selecting from each image in the set. The SEM images spanned magnifications that would typically be used in real particle analyses, $250 x$ to $1000 x$. A set of protocols was established to make the measurements more uniform. The SRM 1984 powder used for these images was subsampled using a riffler, and a sharp-tipped spatula was used to remove the subsample from its sample bottle. The powder was sprinkled on carbon sticky tape mounted on a standard SEM specimen stub.

a. Level of Rigor 1 was designed to quantify the variation that arises from differences in the way independent analysts use the MAMA software on real images.

b. The images were analyzed by a total of 7 different expert MAMA users for this task. The results are discussed in detail in the Uncertainty Determination section.

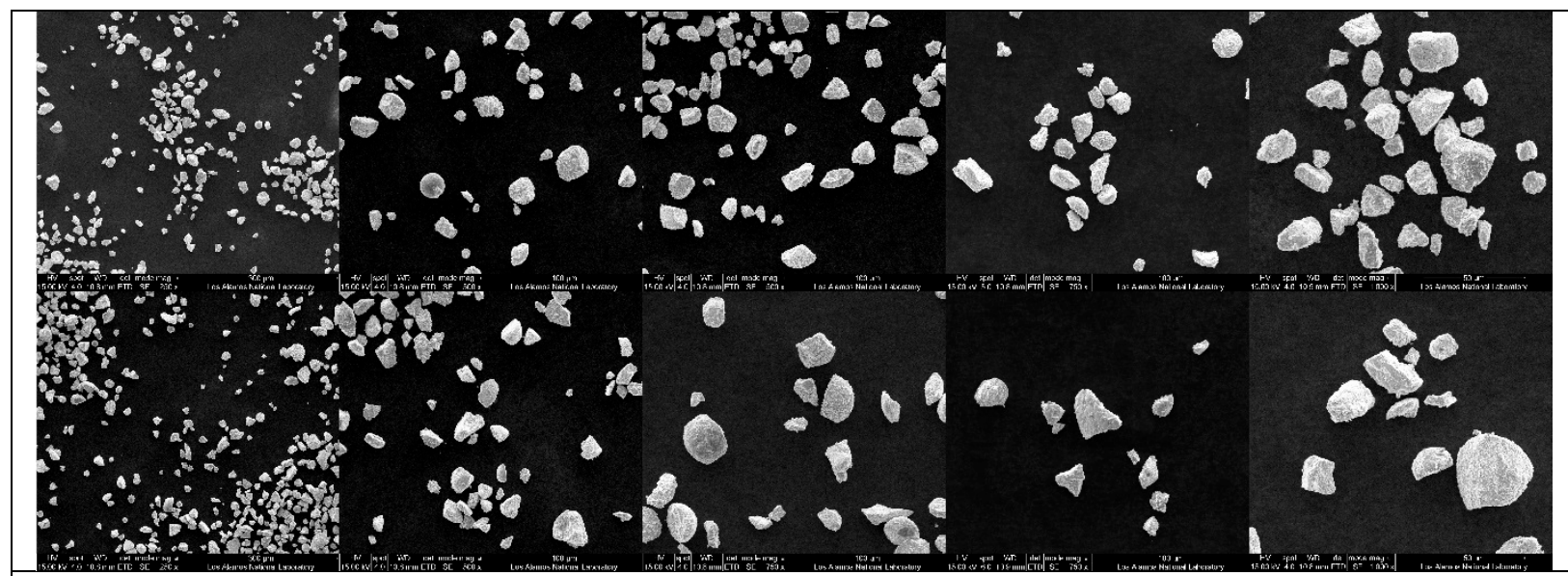

Figure 1. Set of 10 SEM images used in the Level of Rigor 1 round robin exchange.

2. Level of Rigor 2. A set of 4 SEM specimens was made at LANL for distribution to LLNL, PNNL, and SRNL. As in the Level of Rigor 1 test, the SRM 1984 powder for these was subsampled using a 
riffler, and a sharp-tipped spatula was used to remove the subsample from its sample bottle. The powder was sprinkled on carbon sticky tape mounted on a standard SEM specimen stub. The 4 specimens were made at the same time, with an attempt to make them as uniform as possible. The same spatula was used throughout, attempting to get the same level of loading on each SEM mount. The specimens were sent using Fed Ex to the participating laboratories, and no obvious signs of shipping damage were observed. Using SEMs at their laboratory, each participant made a series of images at suitable magnifications and used the MAMA software to measure $>700$ particles.

a. Level of Rigor 2 is designed to quantify uncertainty introduced by differences in the way analysts make SEM images and calibrate their SEMs, in addition to uncertainties associated with Level of Rigor 1.

b. All data from this exchange has been received and processed.

3. Level of Rigor 3. Each laboratory used their own bottle of SRM 1984 to make a mount for SEM examination. LANL, LLNL and SRNL used a riffler to subdivide their SRM 1984, while PNNL used the "cone and quarter" method. Each analyst then made a series of SEM images from their mounts, and used MAMA to analyze the particles in those images.

a. Level of Rigor 3 introduces uncertainties associated with mounting and subsampling powders on top of the uncertainties already captured in the Level of Rigor 1 and 2 exercises.

b. All data from this exchange has been received and processed.

\section{Phase 3 Task 3 Uncertainty Determination}

Uncertainty for Level of Rigor 1.

A total of 7 analysts made measurements using MAMA on the set of 10 SEM images produced at LANL, as described under Task 2. The analysts will be referred to as U1, U2...,U7 ("user 1, 2, etc."). The number of particles measured by each analyst is shown in Table 1 . The third column shows the $95 \%$ confidence interval as a fraction of $\sigma$, the standard deviation of the distribution, based on the usual t-distribution analysis. [Note that this is often expressed as (Mean value) \pm (confidence interval*standard deviation)].

\begin{tabular}{|c|c|c|}
\hline & No. of particles analyzed & Confidence interval, L/ $\sigma$ \\
\hline U1 & 633 & 0.0780 \\
\hline U2 & 624 & 0.0786 \\
\hline U3 & 624 & 0.0786 \\
\hline U4 & 599 & 0.0802 \\
\hline U5 & 487 & 0.0890 \\
\hline U6 & 633 & 0.0781 \\
\hline U7 & 568 & 0.0824 \\
\hline
\end{tabular}


The data was tabulated for each morphological measurement, and a variety of statistical analyses were performed to compare the data sets, which will be described in detail.

It was immediately clear that the U5 data set was a significant outlier, with values for each morphological parameter that were outside of the confidence intervals of all other data sets (probably due to insufficient training on the MAMA software). This can be seen directly by using a binary t-test comparison at $95 \%$ confidence between each data set. Every data set was compared, so there were 21 total binary comparisons. The t-statistic for each comparison is plotted in Figure 2, based on the hypothesis that the datasets have the same mean diameter value.

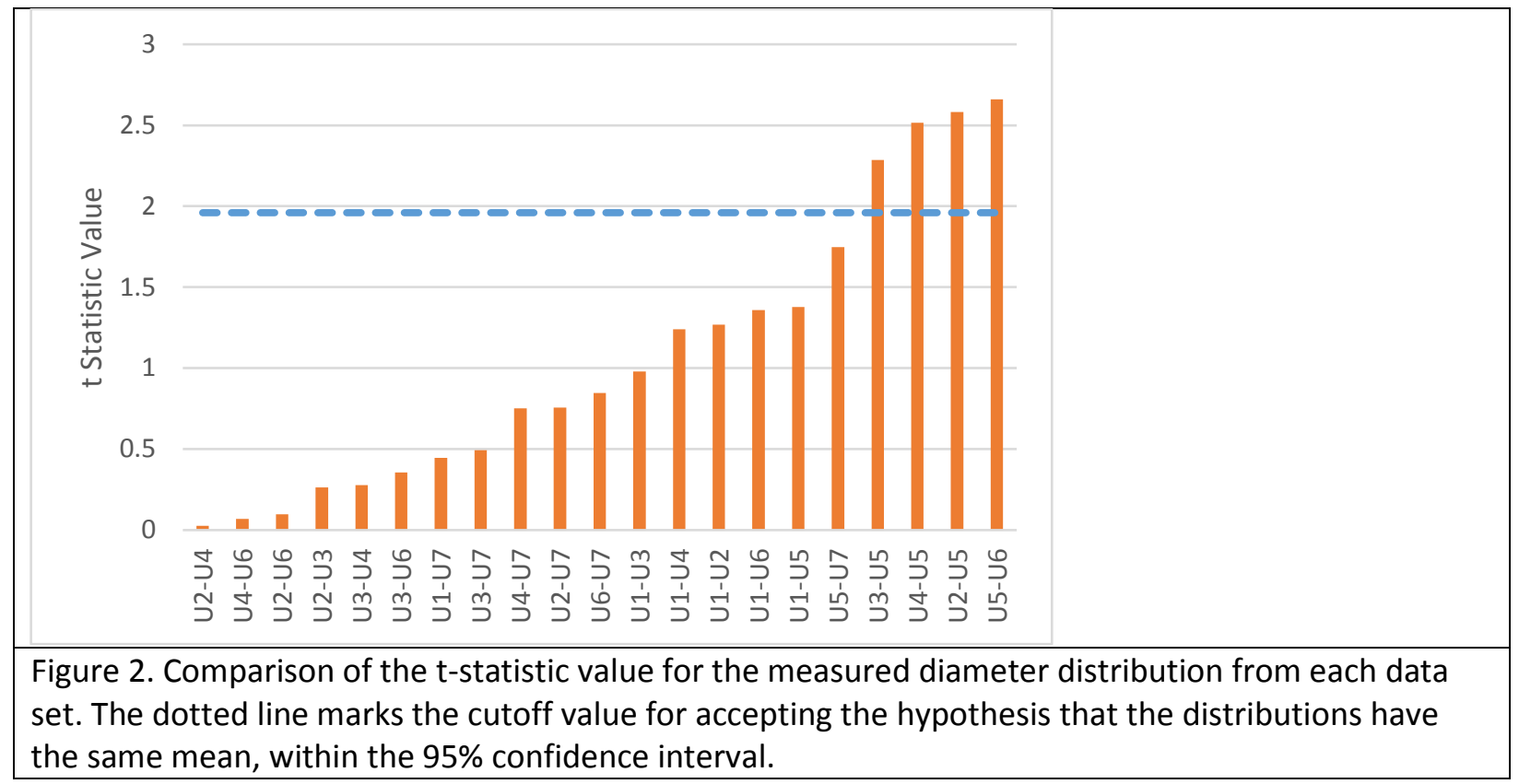

It can be seen in Figure 2 that the U5 data set has the 6 highest t-statistic values (i.e. poorest agreement of the mean diameter), with 4 of the data sets exceeding the limit of t-test acceptance. The poor agreement was observed for all the measured morphological parameters for the U5 dataset, so U5 was declared an outlier and this set was excluded from the statistical analysis.

Three methods were used to quantify the uncertainty involved in using MAMA to measure the morphological parameters of the particles in the LANL image set. They include 1) uncertainty band overlap, 2) histogram analysis, and 3) comparison of cumulative values.

\section{Uncertainty Interval Overlap}

Using a 95\% confidence level (coverage $k=2$ ) we can determine the confidence interval for each measured quantity in the data sets from each user. This standard confidence interval is a function of the number of measurements and the standard deviation of the measured population, and is based on the mathematics of normally distributed random variables. The confidence intervals can be compared across the 6 analysts for each measurement, and the spread between the overall maximum and overall minimum can be determined. This overall spread in values may be regarded as a total confidence band, 
which includes both randomness and differences between how analysts use the MAMA software. The vector area will be used as an example, shown in Table 2.

\begin{tabular}{|c|c|c|}
\hline Analyst & $\begin{array}{c}\text { Mean vector area }\left(\mu \mathrm{m}^{2}\right) \text { from } \\
\text { LANL image set }\end{array}$ & Confidence interval $\left(u_{c}\right)$ \\
\hline U1 & 222.73 & 11.93 \\
\hline $\mathrm{U} 2$ & 213.77 & 10.88 \\
\hline U3 & 216.80 & 11.45 \\
\hline U4 & 218.16 & 11.85 \\
\hline U6 & 213.75 & 10.89 \\
\hline U7 & 219.58 & 12.12 \\
\hline
\end{tabular}

The $95 \%$ confidence interval numbers were then used to plot the spread of the vector area morphological parameter (Figure 3). The error bars in Figure 3 mark the upper and lower limits of the individual $95 \%$ confidence band for each analysts' data. The highest possible value is from U1, U1 $+\mathrm{u}_{\mathrm{c}}=$ $222.73+11.93=234.66$, while the lowest is from U6, U6 - uc $=213.75-10.893=202.86$. The uncertainty interval is the spread between the highest and lowest error bars over the entire set, and is expressed as $202.86<$ vector area $<234.66$. It should be noted that the largest uncertainty spread for a single analysts (U7) was $\sim 24.2 \mu \mathrm{m}^{2}$, while that for the entire group of analysts was $31.8 \mu \mathrm{m}^{2}$, so it is clear that differences in the way expert analysts use MAMA does introduce additional uncertainty. This is termed the "maximum uncertainty" for the morphological parameter.

\begin{tabular}{|l|l|l|}
\hline & Vector Area $\left(\mu \mathrm{m}^{2}\right)$ \\
\hline & \\
&
\end{tabular}


This process of determining 95\% confidence band overlap was performed for the entire data set and is summarized in Table 3. The high and low values for each analysts are the extremes of the 95\% confidence band for their individual data sets.

Note on the "NIST diameter" morphological parameter

The Certificate of Analysis that accompanies the NIST SRM 1984 introduces a diametral morphological parameter that is not commonly used, and requires some explanation. They first fit each particle to an ellipse (as done by the MAMA software), giving a major (A) and minor (B) axis. From these values, they project a prolate ellipsoid, specifically, an ellipsoid with axes $A \neq B=C$. The "NIST diameter" is then found from the average of these three axes: $1 / 3(A+B+C)=1 / 3(A+2 B)$. For typical convex particles, the value of this averaged diameter is quite close to the equivalent circular diameter, $(E C D) \equiv$ $\sqrt{4 / \pi(\text { Area })}$. MAMA does not calculate the NIST diameter as a basic parameter, but it does calculate the major and minor ellipse axes of ellipses fit to each particle, so it is a simple matter to determine the NIST diameter for each data set using Excel or other data handling software.

Although ECD and NIST diameter are similar in magnitude, the two morphological parameters differ in an important way. The NIST diameter is calculated using a complex, multistep mathematical process, involving determining the edge points of a particle, fitting an ellipse to these points, finding the major and minor axes, and finally averaging them. In contrast, the ECD is a simple function of the particle area, which is determined by summing particle pixels. Therefore, comparing the uncertainties for ECD and NIST diameter quantifies the additional uncertainty introduced by fitting computations in MAMA. As seen in Table 3, the uncertainties are slightly different: $1.06 \mu \mathrm{m}$ vs. $1.13 \mu \mathrm{m}$ for the ECD and NIST diameter, respectively.

\begin{tabular}{|l|l|l|l|l|l|l|}
\hline \multicolumn{2}{|l|}{ Table 3. Uncertainties for each MAMA morphological parameter based on confidence band analysis } \\
\hline & $\begin{array}{l}\text { Vector area } \\
\left(\mu \mathrm{m}^{2}\right)\end{array}$ & $\begin{array}{l}\text { Convex hull } \\
\text { area }\left(\mu \mathrm{m}^{2}\right)\end{array}$ & $\begin{array}{l}\text { Pixel area } \\
\left(\mu \mathrm{m}^{2}\right)\end{array}$ & $\begin{array}{l}\text { Vector } \\
\text { perimeter } \\
(\mu \mathrm{m})\end{array}$ & $\begin{array}{l}\text { Convex hull } \\
\text { perimeter } \\
(\mu \mathrm{m})\end{array}$ & $\begin{array}{l}\text { Ellipse } \\
\text { perimeter } \\
(\mu \mathrm{m})\end{array}$ \\
\hline U1 low & 210.80 & 227.41 & 211.52 & 59.80 & 54.40 & 52.70 \\
\hline U1 high & 234.66 & 253.44 & 235.37 & 63.09 & 57.24 & 55.45 \\
\hline U2 low & 202.89 & 215.69 & 203.18 & 57.39 & 53.12 & 51.57 \\
\hline U2 high & 224.65 & 238.48 & 224.93 & 60.21 & 55.68 & 54.10 \\
\hline U3 low & 205.34 & 220.24 & 205.50 & 58.51 & 53.59 & 51.78 \\
\hline U3 high & 228.25 & 245.24 & 228.39 & 61.72 & 56.34 & 54.48 \\
\hline U4 low & 206.30 & 219.25 & 206.29 & 57.71 & 53.08 & 51.41 \\
\hline U4 high & 230.01 & 244.17 & 229.99 & 60.86 & 55.91 & 54.17 \\
\hline U6 low & 202.86 & 217.25 & 202.87 & 58.15 & 53.22 & 51.46 \\
\hline U6 high & 224.64 & 240.34 & 224.64 & 61.10 & 55.84 & 54.02 \\
\hline U7 low & 207.46 & 220.82 & 209.50 & 58.25 & 53.55 & 52.10 \\
\hline U7 high & 231.70 & 246.42 & 238.96 & 61.45 & 56.38 & 55.04 \\
\hline $\begin{array}{l}\text { Maximum } \\
\text { uncertainty }\end{array}$ & 31.80 & 37.75 & 36.09 & 5.70 & 4.15 & 4.03 \\
\hline
\end{tabular}




\begin{tabular}{|l|l|l|l|l|}
\hline $\begin{array}{l}\text { (table } \\
\text { continued) }\end{array}$ & $E C D(\mu \mathrm{m})$ & Major ellipse $(\mu \mathrm{m})$ & Minor ellipse $(\mu \mathrm{m})$ & NIST diameter $(\mu \mathrm{m})$ \\
\hline U1 low & 15.63 & 19.61 & 13.50 & 15.57 \\
\hline U1 high & 16.43 & 20.70 & 14.24 & 16.37 \\
\hline U2 low & 15.41 & 19.14 & 13.29 & 15.26 \\
\hline U2 high & 16.16 & 20.13 & 13.99 & 16.01 \\
\hline U3 low & 15.44 & 19.30 & 13.23 & 15.28 \\
\hline U3 high & 16.23 & 20.37 & 13.96 & 16.07 \\
\hline U4 low & 15.42 & 19.04 & 13.31 & 15.24 \\
\hline U4 high & 16.25 & 20.10 & 14.08 & 16.06 \\
\hline U6 low & 15.38 & 19.08 & 13.28 & 15.24 \\
\hline U6 high & 16.14 & 20.09 & 13.98 & 15.99 \\
\hline U7 low & 15.52 & 19.32 & 13.45 & 15.38 \\
\hline U7 high & 16.36 & 20.46 & 14.26 & 16.20 \\
\hline $\begin{array}{l}\text { Maximum } \\
\text { uncertainty }\end{array}$ & 1.06 & 1.67 & 1.03 & 1.13 \\
\hline
\end{tabular}

\begin{tabular}{|l|l|l|l|l|l|}
\hline $\begin{array}{l}\text { (table } \\
\text { continued) }\end{array}$ & $\begin{array}{l}\text { Ellipse aspect } \\
\text { ratio }\end{array}$ & $\begin{array}{l}\text { Diameter } \\
\text { aspect ratio }\end{array}$ & Circularity & $\begin{array}{l}\text { Perimeter } \\
\text { convexity }\end{array}$ & Area convexity \\
\hline U1 low & 1.46 & 1.41 & 0.67 & 0.91 & 0.92 \\
\hline U1 high & 1.52 & 1.46 & 0.69 & 0.91 & 0.93 \\
\hline U2 low & 1.45 & 1.40 & 0.70 & 0.92 & 0.93 \\
\hline U2 high & 1.50 & 1.45 & 0.72 & 0.93 & 0.94 \\
\hline U3 low & 1.48 & 1.41 & 0.68 & 0.91 & 0.93 \\
\hline U3 high & 1.57 & 1.83 & 0.70 & 0.92 & 0.93 \\
\hline U4 low & 1.44 & 1.39 & 0.70 & 0.92 & 0.93 \\
\hline U4 high & 1.50 & 1.45 & 0.71 & 0.92 & 0.94 \\
\hline U6 low & 1.44 & 1.39 & 0.68 & 0.91 & 0.93 \\
\hline U6 high & 1.50 & 1.45 & 0.70 & 0.92 & 0.93 \\
\hline U7 low & 1.44 & 1.40 & 0.69 & 0.92 & 0.93 \\
\hline U7 high & 1.50 & 1.46 & 0.71 & 0.92 & 0.94 \\
\hline $\begin{array}{l}\text { Maximum } \\
\text { uncertainty }\end{array}$ & 0.12 & 0.43 & 0.05 & 0.02 & 0.02 \\
\hline
\end{tabular}

$\underline{\text { Histogram analysis }}$

A second approach to determining uncertainty is to compare the data sets in histogram form. This methodology is necessarily less precise, because it is based on binning the data into relatively coarse bins. However, it is the only method that can quantify uncertainty in the shape of the distribution of values for a morphological parameter. This methodology will be demonstrated for the NIST diameter 
and Pixel Area morphological parameters (as described in the previous section, these two parameters are of different types, one highly calculated and the other very basic).

The data sets for each analyst, excluding the U5 outlier, are shown in histogram form in Figure 4. The advantage of this type of plot is that the shape of the entire population distribution is apparent. The shape is itself a characteristic of the population, separate from the statistical values that can be calculated from the data set, such as mean, standard deviation, etc. The amount of variation within each bin can be clearly seen by putting all the analysts' data into a single plot, as done in Figure 4 .

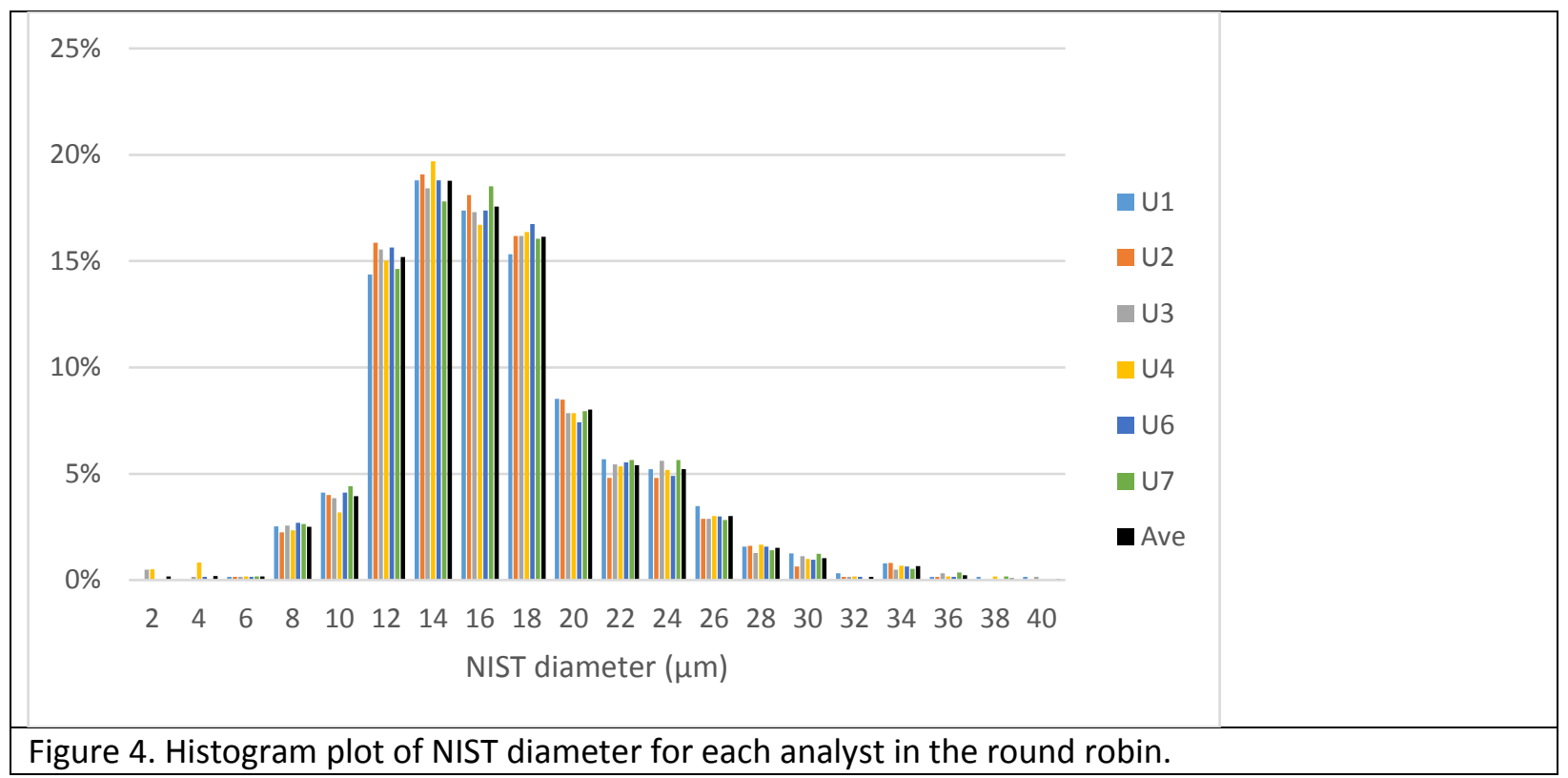

The variation can be quantified by finding the average and standard deviation for each bin. This is shown in Figure 5, and it can be seen that the bins are tightly defined, i.e. the variation between the analysts' data within the bins is small. The variation can be quantified as a percentage of the bin, which is tabulated in Table 4 for the most populated (central) bins.

The interpretation of these percentage variation values requires explanation. Choosing the $10-12 \mu \mathrm{m}$ bin as an example, we see a variation of $3.6 \%$ in Table 4 . This means that for a new data set of similar size, we can expect $3.6 \%$ of the particles that should fall into the $10-12 \mu \mathrm{m}$ bin to either be too small or too large to fall into this bin range. The percentage variation is not an uncertainty in the measured morphological parameter, rather an uncertainty in the fraction of particles that fall into a given size range, and therefore an uncertainty in the shape of the distribution. 


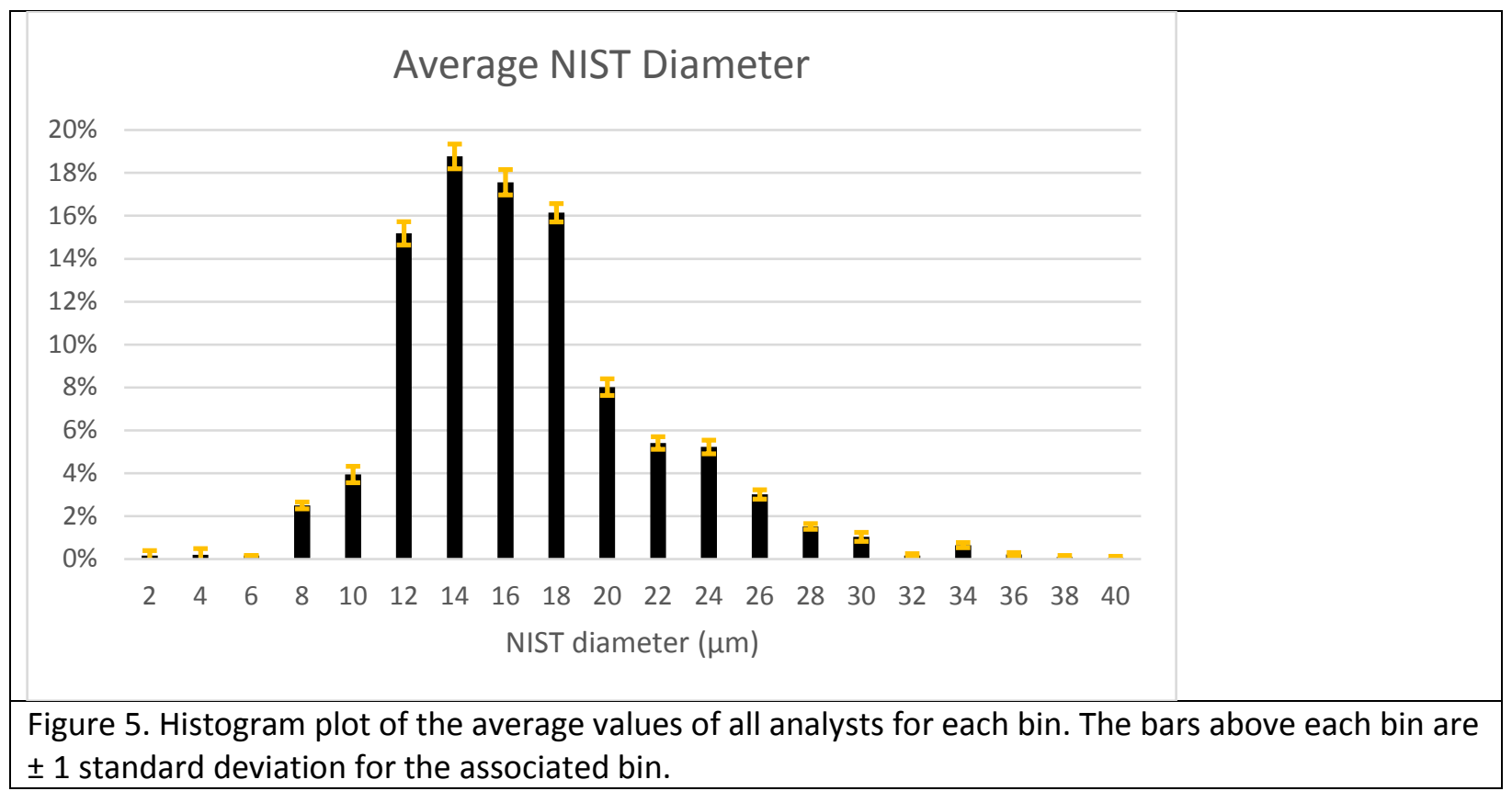

Table 4. Histogram analysis for NIST diameter (central bins)

\begin{tabular}{|c|c|}
\hline Bin $(\mu \mathrm{m})$ & $1-\sigma$ variation within bin \\
\hline $10-12$ & $3.6 \%$ \\
\hline $12-14$ & $3.1 \%$ \\
\hline $14-16$ & $3.4 \%$ \\
\hline $16-18$ & $2.6 \%$ \\
\hline
\end{tabular}

The analysis was repeated for the Pixel Area morphological parameter, as this is the least calculated parameter in the MAMA set. It is a simple sum of all the pixels in a given particle, times the pixels/ $\mu \mathrm{m}$ magnification factor. The histogram in Figure 6 shows the distribution for the Pixel Area, averaged over all the analysts' datasets, with error bars marking one standard deviation above and below the mean for each bin. Table 5 shows the standard deviation as a percent for the four central bins in the distribution. Despite the somewhat different shape of the distribution, the deviation fractions are quite similar to those found for the NIST diameter. 


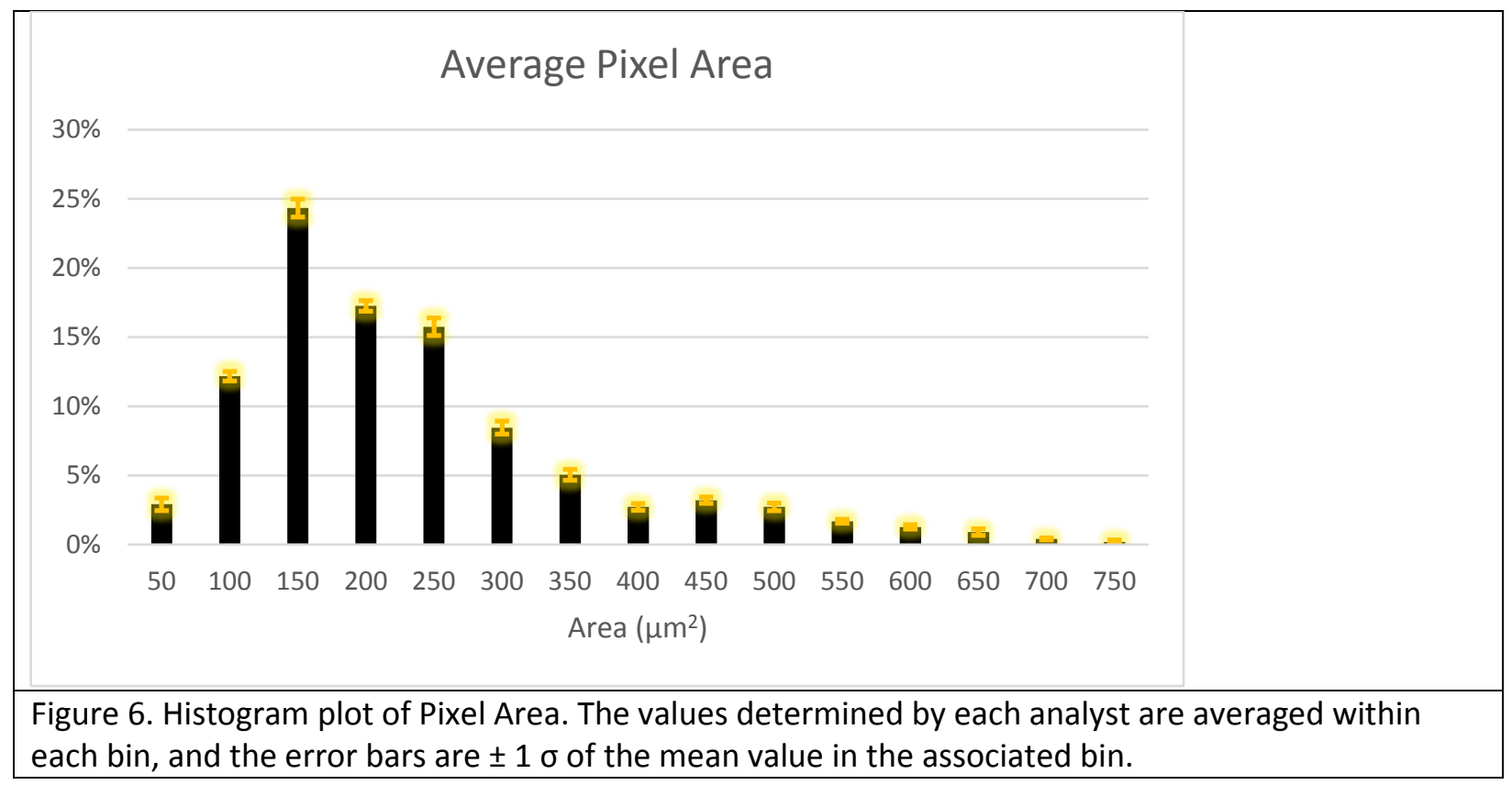

\begin{tabular}{|c|c|}
\hline \multicolumn{2}{|c|}{ Table 5. Percent error within central bins for Pixel Area } \\
\hline Bin $\left(\mu \mathrm{m}^{2}\right)$ & 1- $\sigma$ variation within bin \\
\hline $50-100$ & $2.8 \%$ \\
\hline $100-150$ & $2.7 \%$ \\
\hline $150-200$ & $2.2 \%$ \\
\hline $200-250$ & $4.0 \%$ \\
\hline
\end{tabular}

Analysis of cumulative frequency plots for uncertainty

The cumulative frequency plot is simply the integrated population distribution for a given morphological parameter. The data sets from each analyst are plotted cumulatively in Figure 7, showing the spread of the data. As an example of the use of this type of plot, if we choose $10 \mu \mathrm{m}$ as a target particle diameter, we can read from the $y$-axis that $\sim 7 \%$ of the particles in the population have this diameter or less (follow the blue arrows in Figure 7). It is conventional to report the size fractions at $10 \%, 25 \%, 50 \%$ (the median value), $75 \%$, and $90 \%$. Examination of Figure 7 shows that at each of these values, there is a difference between the analysts. Using a method analogous to the confidence band analysis, we can determine the spread from minimum to maximum and this will be the overall uncertainty for that particular fractional value. 


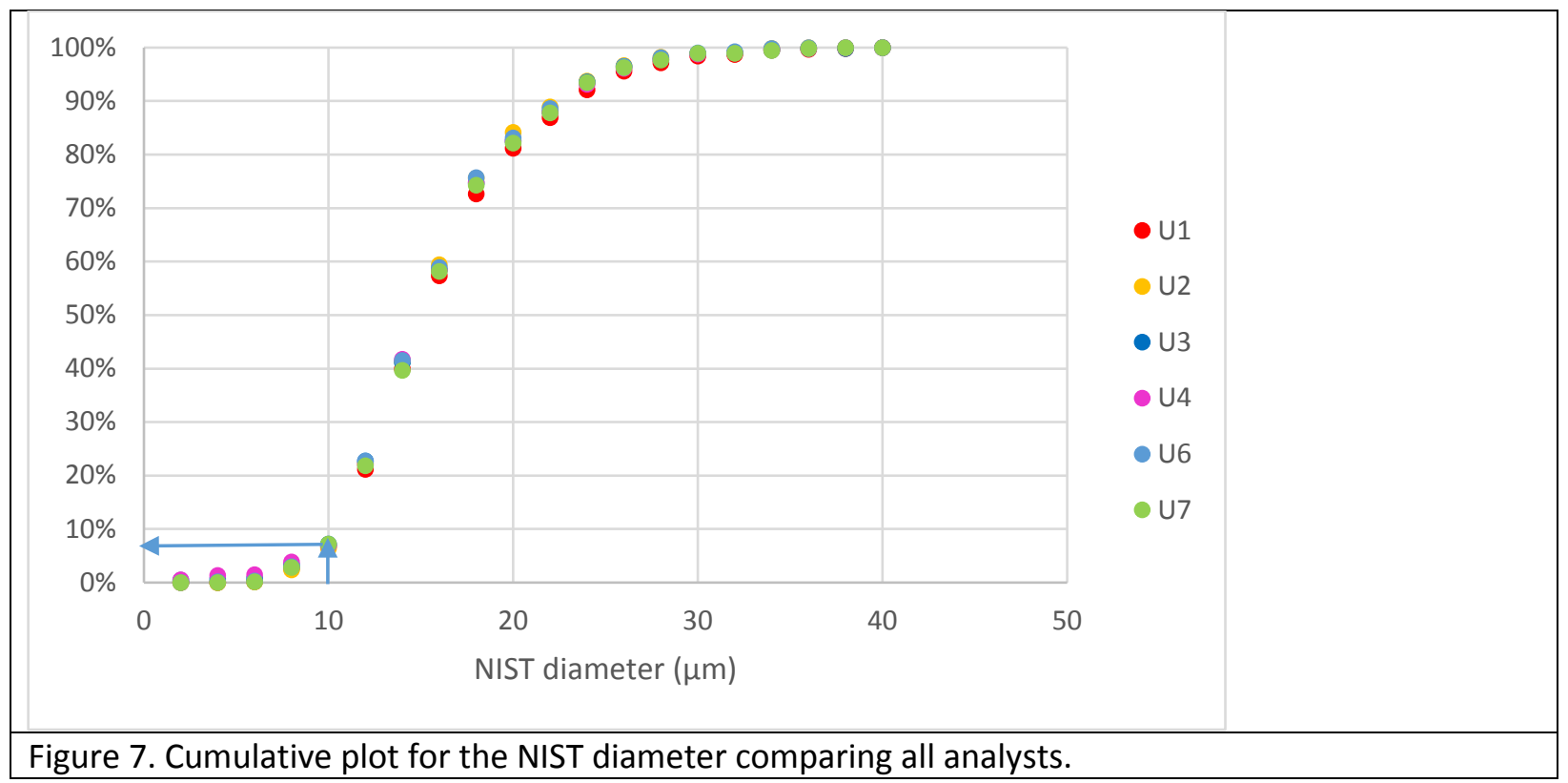

Figure 8 shows the methodology for determining the uncertainty at the $10 \%$ frequency level as an example. It is desired to find the diameter for which $10 \%$ of the population has a smaller value. Comparing the data sets, we want to find the maximum and minimum values that cross the $10 \%$ axis (marked with an ellipse in Figure 8). A simple linear fit was used to calculate the $10 \%$ intercept points, shown in Figure 8 . At the $10 \%$ level, analyst U1 had the largest value $(10.44 \mu \mathrm{m})$, while analyst U6 has the lowest value $(10.37 \mu \mathrm{m})$. Therefore the uncertainty at the $10 \%$ frequency is $\sim 0.07 \mu \mathrm{m}$. The values at standard cumulative frequency points, and the maximum spread of values is tabulated in Table 6 . The same calculation was performed for the pixel area morphological parameter and the results are shown in Table 7.

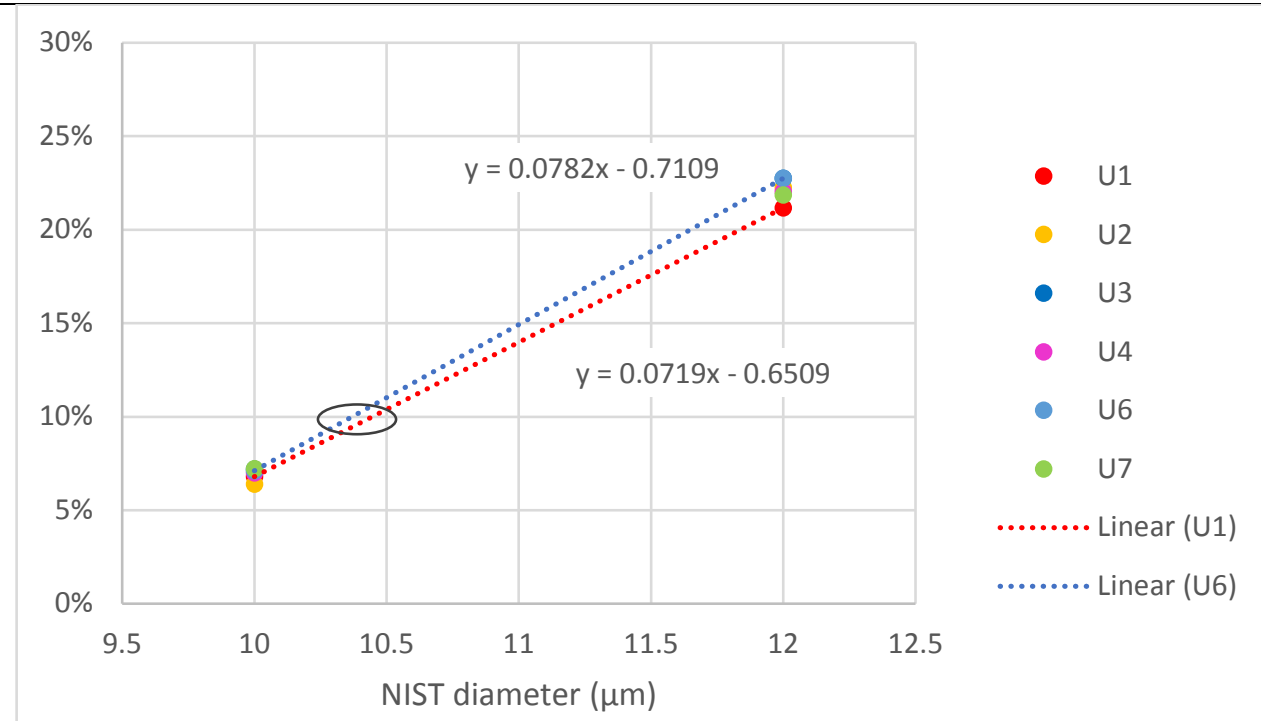


Figure 8. Methodology for determining uncertainty at $10 \%$ frequency level (see text).

\begin{tabular}{|c|c|c|c|}
\hline \multicolumn{4}{|c|}{ Table 6. Spread of NIST diameter values at selected cumulative frequency regions. } \\
\hline & Maximum value & Minimum value & Uncertainty \\
\hline $10 \%$ & 10.44 & 10.37 & 0.07 \\
\hline $25 \%$ & 12.41 & 12.24 & 0.17 \\
\hline $50 \%$ (median) & 15.15 & 14.96 & 0.19 \\
\hline $75 \%$ & 18.53 & 17.93 & 0.60 \\
\hline $90 \%$ & 23.16 & 22.48 & 0.69 \\
\hline
\end{tabular}

Table 7. Spread of pixel area values at selected cumulative frequency regions.

\begin{tabular}{|c|c|c|c|}
\hline & Maximum value & Minimum value & Uncertainty \\
\hline $10 \%$ & 83.08 & 77.16 & 5.92 \\
\hline $25 \%$ & 120.74 & 118.85 & 1.89 \\
\hline $50 \%$ (median) & 182.38 & 176.28 & 6.10 \\
\hline $75 \%$ & 276.71 & 263.94 & 12.76 \\
\hline $90 \%$ & 433.67 & 428.14 & 5.52 \\
\hline
\end{tabular}

Level of Rigor 1 Conclusions

In general, uncertainty introduced by differences in the way analysts use the MAMA software are small. Comparing the NIST diameter in Table 3 and Table 6 shows that the uncertainty determined using the $95 \%$ confidence band is significantly larger than the cumulative plot results (1.13 $\mu \mathrm{m}$ vs. $0.19 \mu \mathrm{m})$. This is also observed for the pixel area, cf. Table 3 and Table $7\left(36.09 \mu \mathrm{m}^{2}\right.$ vs. $\left.6.1 \mu \mathrm{m}^{2}\right)$. The $95 \%$ confidence band analysis will always yield a larger calculated uncertainty as it includes both uncertainty in the measurements due to random statistical errors and differences between users. The other methods for quantifying uncertainty, histogram analysis and cumulative frequency analysis, only measure differences between users MAMA analyses. 
Uncertainty in the use of MAMA software to measure particle morphological parameters from SEM images

\section{Part 2: Special Tests and Level of Rigor 2 and 3 uncertainty}

Phase 3 Task 3 Uncertainty Determination

Special MAMA analysis tests

Based on MAMA user feedback and observations from the LANL MAMA expert user group, a series of focused investigations were undertaken to test specific significantly important aspects of the software. The results of these investigations have been incorporated into the MAMA usage protocol document (Appendix A) developed in this project and will be described in detail below.

1. Image Magnification. The protocol document calls for the use of a magnification calibration standard to determine image magnification. However, the majority of MAMA users simply use the MAMA ruler tool to set the magnification. The ruler tool uses a click and drag routine to measure the size bar in the image being analyzed, and therefore introduces error into the image magnification. This error propagates through all the morphological quantifications, except for those based on ratios (e.g. aspect ratio and circularity).

Results from Phase 3, Task 1 were compared to determine how much variation there was between users in the magnifications they determined using the MAMA ruler tool. Six different analysts used the MAMA ruler to quantify the magnification for 10 SEM images supplied by LANL for the round robin effort. The standard deviations of the magnifications for each image were determined and are plotted in Figure 1 . All errors were $<0.25 \%$, and the overall average error was found to be $0.14 \%$. These errors are insignificant compared to the larger errors introduced by differences in segmentation and can be neglected. However, the calibration of the SEM image size bar cannot be neglected, and is an important part of the quantification process (see the protocol document in Appendix A). 


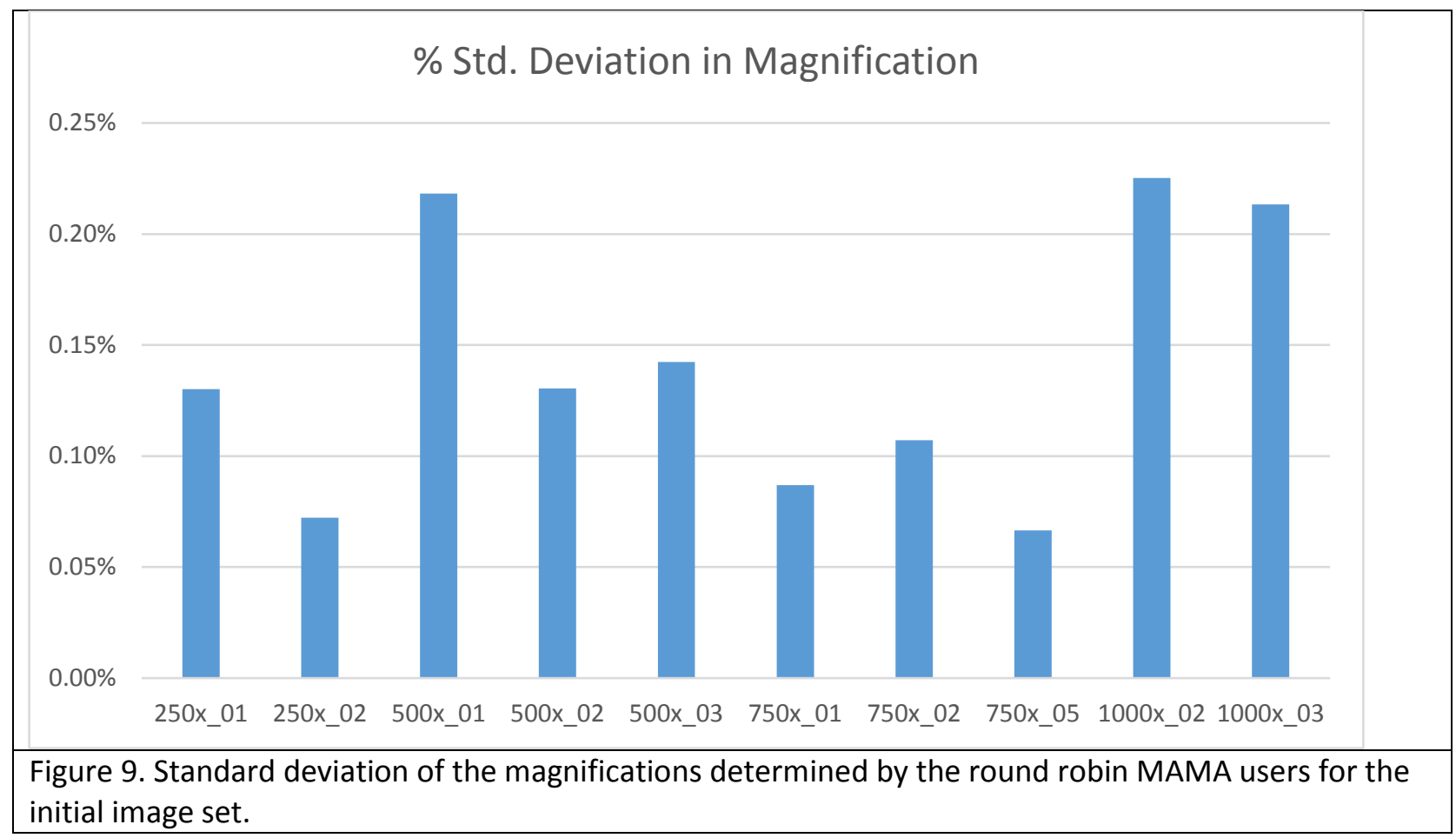

2. Segmentation filter effects. MAMA 2.0.2 allows the user to select from 6 different preset segmentation filters, and to create unlimited custom filters. It is important to point out and quantify the large effect these filters have on the quantification of morphological parameters. A simple comparison was made by using the "smoothest" and "detailest" filters on a set of 7 typical particles. These filters represent two extremes in the way MAMA defines the edge of an object: the smoothest filter uses a series of techniques to find an average edge, with asperities in the perimeter smoothed out. If we compare the measured pixel area for each particle (Figure 2 ) we observe that the difference between the two filters can be as high as $\sim 1 \%$. There is a clear trend toward smaller errors for larger particles, but there is also an effect due to shape of the particle. Particles with a more convex shape show less difference between the filters. Morphological parameters that involve perimeter measurements show a large difference between the two filters. The difference in vector perimeter as a function of number of particle pixels is plotted in Figure 3, and differences approach 7\%. In addition, it is clear that the smoothest filter always results in a smaller vector perimeter (as expected for a smoothed object edge). The differences for vector perimeter become smaller for larger particles, but remain significant at all size ranges. These errors are not negligible, and it is therefore highly important that MAMA users report the segmentation filters that they use in their analyses. In the protocol document, we recommend using the "smoother" filter as a good compromise filter, but ultimately the choice of filter depends on the morphology of the particles being measured as well as the purpose of the measurements. 

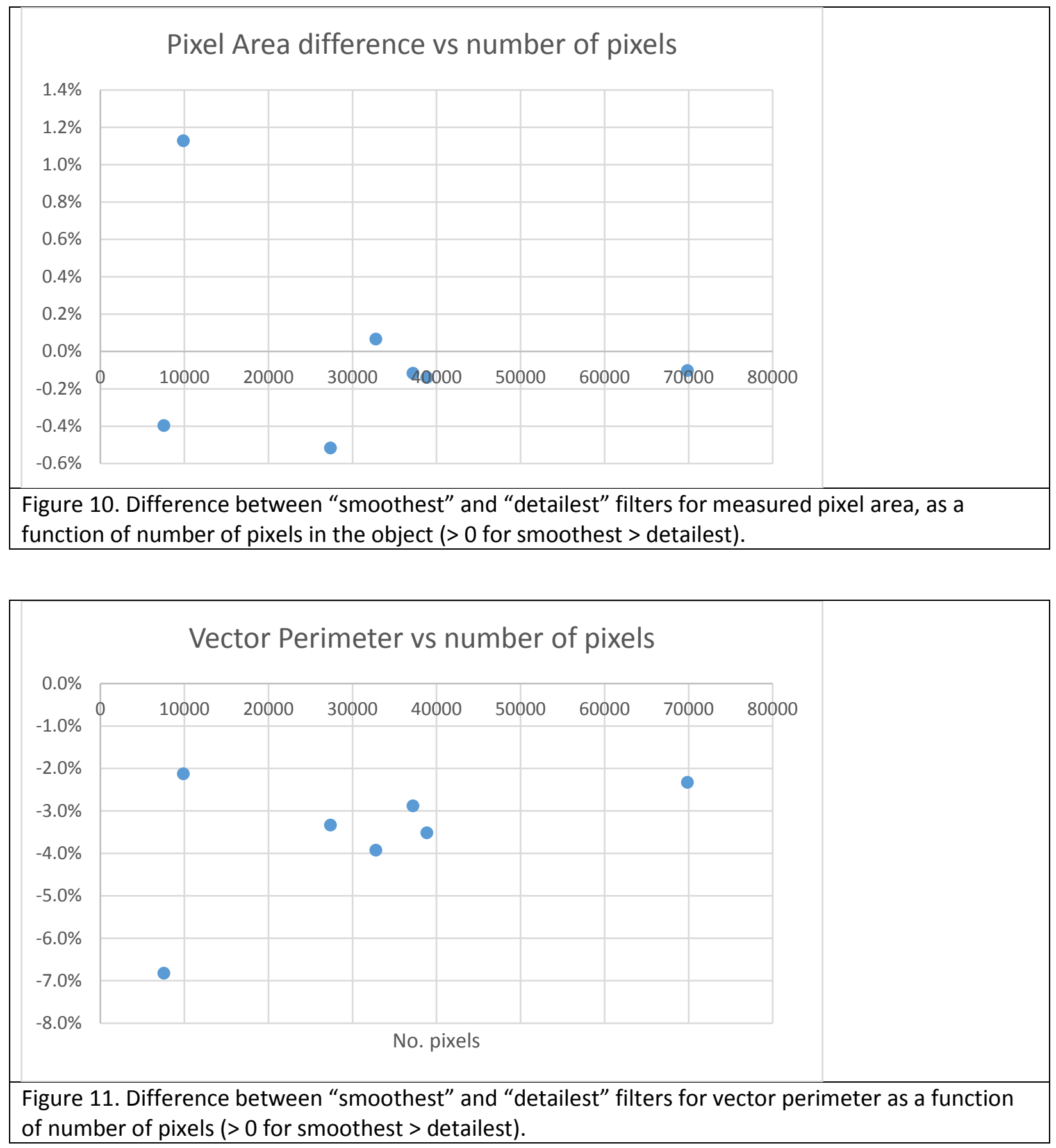

3. Effect of image pixelation: Typically, digital images produced by SEM contain a minimum of $\sim 410^{6}$ pixels. The image of a specific small particle will therefore contain a far lower number of pixels, depending upon the image magnification. The effect of imaging an object with a low number of pixels is clearly visible in Figure 4, where the same particle is imaged at 4 different magnifications and displays increasing pixelation. MAMA was used to measure key morphological parameters to quantify the effects of pixelation. Similar tests have been done 
previously on synthetic objects, but using actual particle images is a more rigorous and useful test.

A set of 7 particles was imaged at magnifications ranging from $250 x$ to $2000 x$, so that the particle images had a wide range of numbers of pixels. MAMA was used to measure morphological parameters, and the values were compared to the 2000x image, which was treated as the most accurate reference measurement. The "smoother" segmentation filter was used. Figure 5 compares the results for the ECD and NIST diameters, and we can see that differences become small for particles above $\sim 1000$ pixels. Pixelation errors depend on the particle shape, as well as number of pixels, so the decrease in error with increasing numbers of pixels is not strictly monotonic. Pixelation does not affect all morphological measurements in the same way, as shown in Figure 6, where the pixel area error is plotted as a function of number of pixels. Errors due to pixelation do not become insignificant until particles have $~ 10^{4}$ pixels for this morphological parameter. Perimeter morphological measurements are very sensitive to pixelation. In particular, the vector perimeter shows large errors for pixelated particles (Figure 7). It is better to use a simpler morphological parameter for perimeter for such particles, like the convex perimeter. As a rule of thumb, we recommend at least 1000 pixels/particle, and it is clear that more is better. For particle populations that contain significant numbers of small particles, it is important to use magnifications high enough to capture the smallest particles with enough pixels to make meaningful measurements.
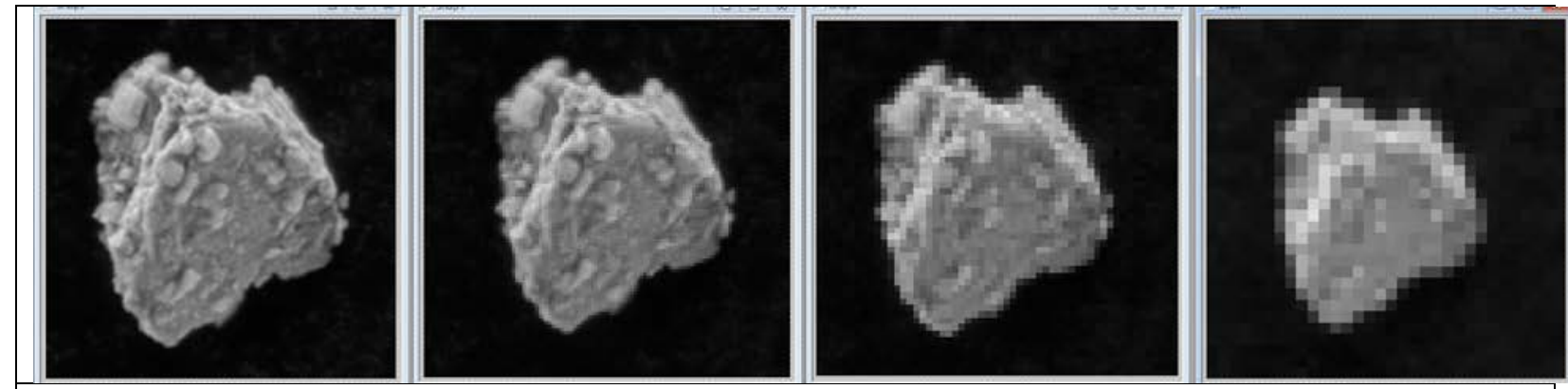

Figure 12. Demonstration of pixelation on a typical particle. Magnification 2000x, 1000x, 500x, and 250x (left to right). 

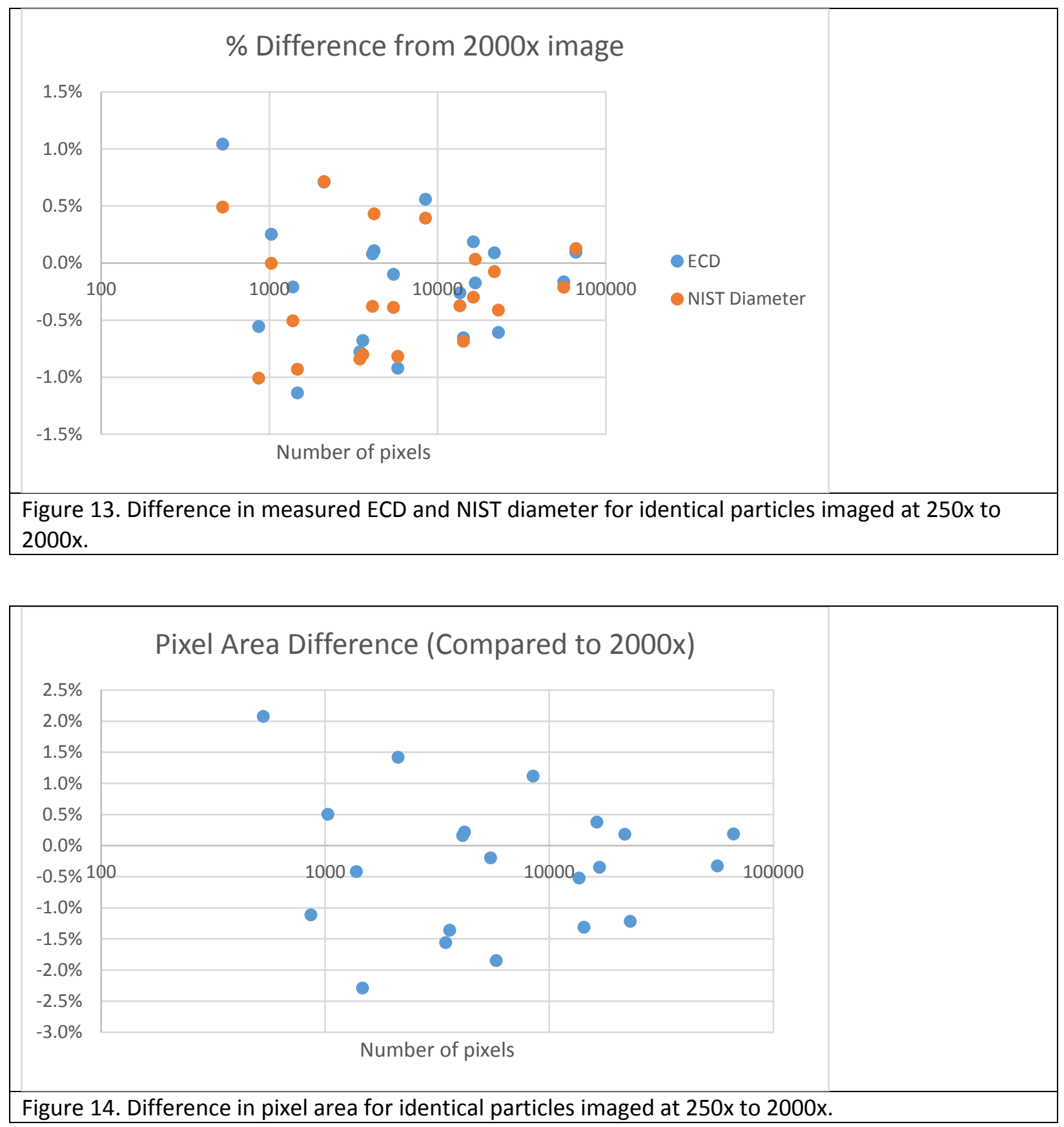


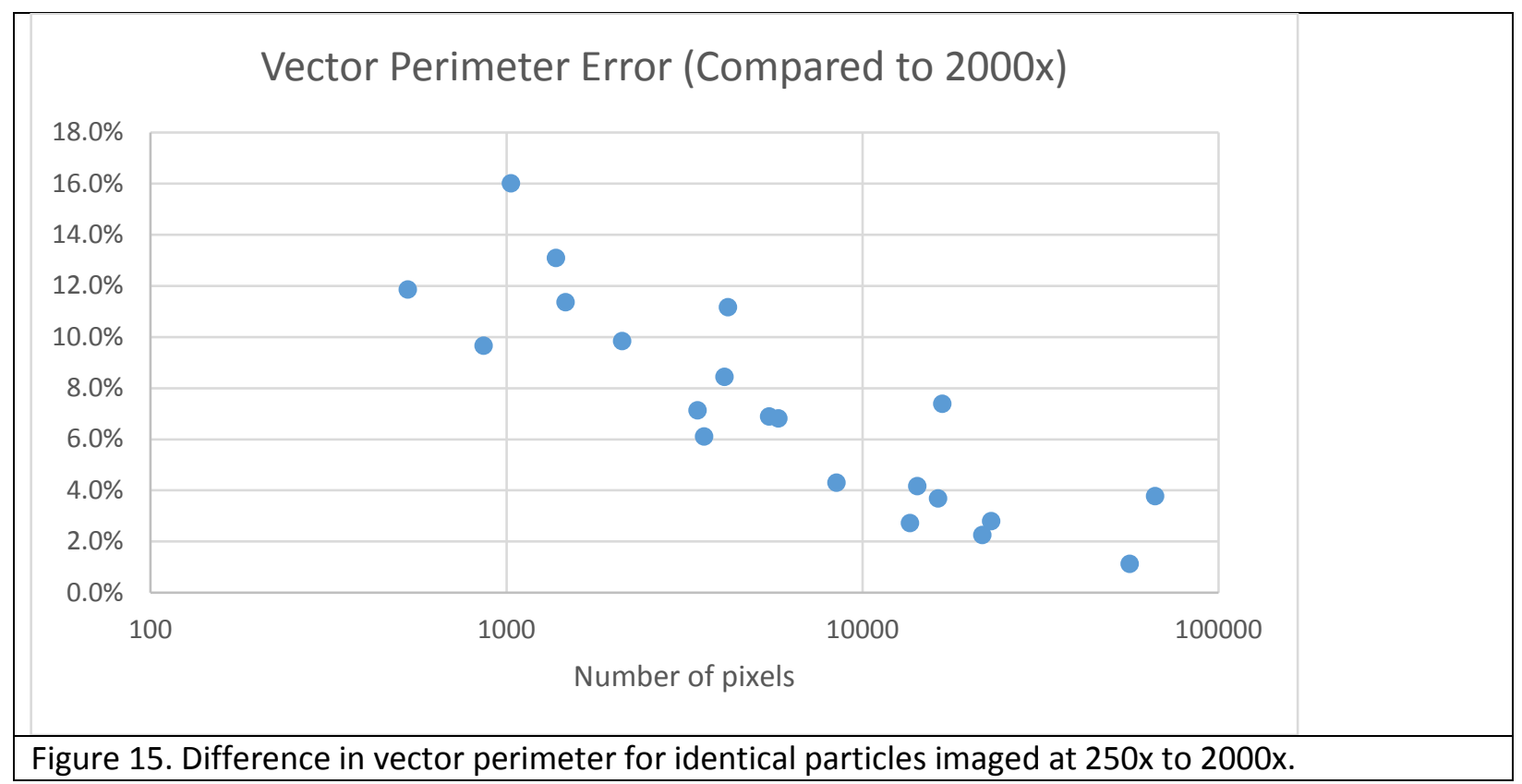

4. "Redo" test: As a basic check of repeatability, three SEM images from the set used for the Level of Rigor 1 round robin exercise were remeasured and the results compared. Care was taken to treat these efforts as new measurements, with no reference to the original measurements. The original measurements were made roughly one year prior to the redo, so the analyst had no significant memory of the first measurements.

The redone measurements contained 140 particles, compared to 136 for the original measurements, demonstrating that even the same expert user analyzing the same images will not always make exactly the same decisions about which particles to include in the analysis. However, the measured morphological parameters were quite similar. Table 1 compares the original values to the redone analysis for key statistical values from each measurement set. The match for all mean values is quite good, and it should be noted that the differences are 1 order of magnitude below the $95 \%$ confidence band size. Kurtosis and skew match less well, but these parameters are very sensitive, particularly at relatively low population numbers.

\begin{tabular}{|c|c|c|c|c|c|c|}
\hline \multicolumn{2}{|l|}{ Table 8. Comparison of statistical values for the "redo" test. } \\
\hline & ORIGINAL & REDO & \multicolumn{2}{|c|}{ ORIGINAL } & REDO & \multicolumn{2}{l|}{ ORIGINAL } & REDO \\
\hline & \multicolumn{2}{|c|}{ Vector area $\left(\mu \mathrm{m}^{2}\right)$} & \multicolumn{2}{c|}{ Convex hull area $\left(\mu \mathrm{m}^{2}\right)$} & \multicolumn{2}{|c|}{ Pixel area $\left(\mu \mathrm{m}^{2}\right)$} \\
\hline Mean & 223.09 & 219.07 & 235.93 & 232.48 & 223.08 & 219.06 \\
\hline Standard Error & 14.59 & 14.37 & 15.13 & 15.03 & 14.59 & 14.37 \\
\hline Median & 179.23 & 174.69 & 190.22 & 188.17 & 179.23 & 174.68 \\
\hline $\begin{array}{c}\text { Standard } \\
\text { Deviation }\end{array}$ & 170.17 & 170.05 & 176.45 & 177.81 & 170.17 & 170.05 \\
\hline Kurtosis & 3.19 & 3.06 & 3.08 & 2.84 & 3.19 & 3.06 \\
\hline Skewness & 1.60 & 1.58 & 1.56 & 1.54 & 1.60 & 1.58 \\
\hline
\end{tabular}




\begin{tabular}{|c|c|c|c|c|c|c|}
\hline Minimum & 34.88 & 34.69 & 36.90 & 36.64 & 34.88 & 34.69 \\
\hline Maximum & 931.63 & 929.08 & 973.81 & 971.35 & 931.61 & 929.05 \\
\hline Sum & 30340.55 & 30670.15 & 32086.82 & 32547.82 & 30339.24 & 30668.27 \\
\hline $\begin{array}{c}95 \% \\
\begin{array}{c}\text { Confidence } \\
\text { Level }\end{array}\end{array}$ & 28.86 & 28.42 & 29.92 & 29.71 & 28.86 & 28.42 \\
\hline
\end{tabular}

\begin{tabular}{|c|c|c|c|c|c|c|}
\hline Table 1 cont'd & ORIGINAL & REDO & ORIGINAL & REDO & ORIGINAL & REDO \\
\hline & \multicolumn{2}{|c|}{ Vector perimeter $(\mu \mathrm{m})$} & \multicolumn{2}{c|}{ Convex hull perim $(\mu \mathrm{m})$} & \multicolumn{2}{c|}{ Ellipse perimeter $(\mu \mathrm{m})$} \\
\hline Mean & 59.59 & 59.20 & 54.14 & 53.71 & 52.40 & 51.99 \\
\hline Standard Error & 1.88 & 1.91 & 1.69 & 1.71 & 1.65 & 1.68 \\
\hline Median & 57.96 & 57.37 & 52.33 & 51.83 & 50.60 & 50.00 \\
\hline $\begin{array}{c}\text { Standard } \\
\text { Deviation }\end{array}$ & 21.96 & 22.65 & 19.73 & 20.20 & 19.29 & 19.86 \\
\hline Kurtosis & -0.01 & 0.29 & 0.01 & 0.10 & 0.03 & 0.24 \\
\hline Skewness & 0.59 & 0.75 & 0.61 & 0.71 & 0.63 & 0.76 \\
\hline Minimum & 24.37 & 24.34 & 22.38 & 22.31 & 21.70 & 21.62 \\
\hline Maximum & 127.98 & 128.72 & 114.69 & 114.53 & 111.37 & 111.31 \\
\hline Sum & 8104.41 & 8288.02 & 7363.46 & 7519.40 & 7126.45 & 7278.07 \\
\hline $\begin{array}{c}\text { 95\% } \\
\text { Confidence } \\
\text { Level }\end{array}$ & 3.72 & & & & & \\
\hline
\end{tabular}

\begin{tabular}{|c|c|c|c|c|c|c|}
\hline Table 1 cont'd & ORIGINAL & REDO & ORIGINAL & REDO & ORIGINAL & REDO \\
\hline & \multicolumn{2}{|c|}{$E C D(\mu m)$} & \multicolumn{2}{|c|}{ Major ellipse $(\mu \mathrm{m})$} & \multicolumn{2}{|c|}{ Minor ellipse $(\mu \mathrm{m})$} \\
\hline Mean & 15.78 & 15.60 & 19.41 & 19.31 & 13.61 & 13.42 \\
\hline $\begin{array}{c}\text { Standard } \\
\text { Error }\end{array}$ & 0.51 & 0.50 & 0.61 & 0.64 & 0.47 & 0.47 \\
\hline Median & 15.11 & 14.91 & 18.95 & 18.59 & 12.89 & 12.46 \\
\hline $\begin{array}{l}\text { Standard } \\
\text { Deviation }\end{array}$ & 5.93 & 5.97 & 7.16 & 7.58 & 5.53 & 5.52 \\
\hline Kurtosis & 0.18 & 0.11 & -0.13 & 1.53 & 0.94 & 1.00 \\
\hline Skewness & 0.71 & 0.73 & 0.53 & 0.95 & 0.97 & 0.99 \\
\hline Minimum & 6.66 & 6.64 & 7.75 & 7.75 & 5.91 & 4.71 \\
\hline Maximum & 34.44 & 34.39 & 38.60 & 51.21 & 32.16 & 32.12 \\
\hline Sum & 2146.32 & 2184.56 & 2639.49 & 2704.08 & 1851.38 & 1878.78 \\
\hline $\begin{array}{c}95 \% \\
\text { Confidence } \\
\text { Level }\end{array}$ & 1.01 & 1.00 & 1.21 & 1.27 & 0.94 & 0.92 \\
\hline
\end{tabular}

\begin{tabular}{|c|c|c|c|c|c|c|}
\hline $\begin{array}{l}\text { Table } 1 \\
\text { cont'd }\end{array}$ & ORIGINAL & REDO & ORIGINAL & REDO & ORIGINAL & REDO \\
\hline & \multicolumn{2}{|c|}{ Ellipse aspect ratio } & \multicolumn{2}{|c|}{ Diameter aspect ratio } & \multicolumn{2}{|c|}{ Circularity } \\
\hline
\end{tabular}




\begin{tabular}{|c|c|c|c|c|c|c|}
\hline Mean & 1.47 & 1.49 & 1.41 & 1.42 & 0.69 & 0.68 \\
\hline $\begin{array}{c}\text { Standard } \\
\text { Error }\end{array}$ & 0.03 & 0.03 & 0.03 & 0.03 & 0.01 & 0.01 \\
\hline Median & 1.41 & 1.41 & 1.37 & 1.37 & 0.70 & 0.69 \\
\hline $\begin{array}{c}\text { Standard } \\
\text { Deviation }\end{array}$ & 0.34 & 0.38 & 0.30 & 0.32 & 0.07 & 0.07 \\
\hline Kurtosis & 3.01 & 8.16 & 2.46 & 5.39 & 0.89 & 3.06 \\
\hline Skewness & 1.50 & 2.27 & 1.32 & 1.84 & -0.53 & -0.94 \\
\hline Minimum & 1.01 & 1.01 & 1.01 & 1.01 & 0.44 & 0.34 \\
\hline Maximum & 2.82 & 3.57 & 2.63 & 2.90 & 0.86 & 0.86 \\
\hline Sum & 200.00 & 207.95 & 192.28 & 199.37 & 93.73 & 95.75 \\
\hline $\begin{array}{c}\text { 95\% } \\
\text { Confidence } \\
\text { Level }\end{array}$ & 0.06 & 0.06 & 0.05 & & & \\
\hline
\end{tabular}

\begin{tabular}{|c|c|c|c|c|c|c|}
\hline Table 1 cont'd & ORIGINAL & REDO & ORIGINAL & REDO & ORIGINAL & REDO \\
\hline & \multicolumn{2}{|c|}{ Perim convexity } & \multicolumn{2}{|c|}{ Area convexity } & \multicolumn{2}{|c|}{ NIST D3 } \\
\hline Mean & 0.91 & 0.91 & 0.94 & 0.93 & 15.54 & 15.38 \\
\hline $\begin{array}{c}\text { Standard } \\
\text { Error }\end{array}$ & 0.00 & 0.00 & 0.00 & 0.00 & 0.50 & 0.50 \\
\hline Median & 0.91 & 0.91 & 0.94 & 0.94 & 14.94 & 14.66 \\
\hline $\begin{array}{l}\text { Standard } \\
\text { Deviation }\end{array}$ & 0.02 & 0.02 & 0.03 & 0.03 & 5.84 & 5.91 \\
\hline Kurtosis & 6.42 & 1.07 & 5.50 & 5.69 & 0.31 & 0.23 \\
\hline Skewness & -1.66 & -0.79 & -1.72 & -1.70 & 0.74 & 0.77 \\
\hline Minimum & 0.78 & 0.83 & 0.78 & 0.78 & 6.55 & 6.53 \\
\hline Maximum & 0.95 & 0.95 & 0.99 & 0.99 & 34.31 & 34.26 \\
\hline Sum & 123.60 & 127.03 & 127.37 & 130.85 & 2114.08 & 2153.88 \\
\hline $\begin{array}{c}95 \% \\
\text { Confidence } \\
\text { Level }\end{array}$ & 0.00 & 0.00 & 0.00 & 0.00 & 0.99 & 0.99 \\
\hline
\end{tabular}

The data can also be compared using a histogram, as in Figure 8. Three representative morphological parameters were compared, the NIST diameter, vector area and ellipse perimeter. The distributions compare quite accurately, with most bins identical or varying by \pm 1 particle. The redo test establishes that the repeatability of the measurements is good and is a negligible error. 


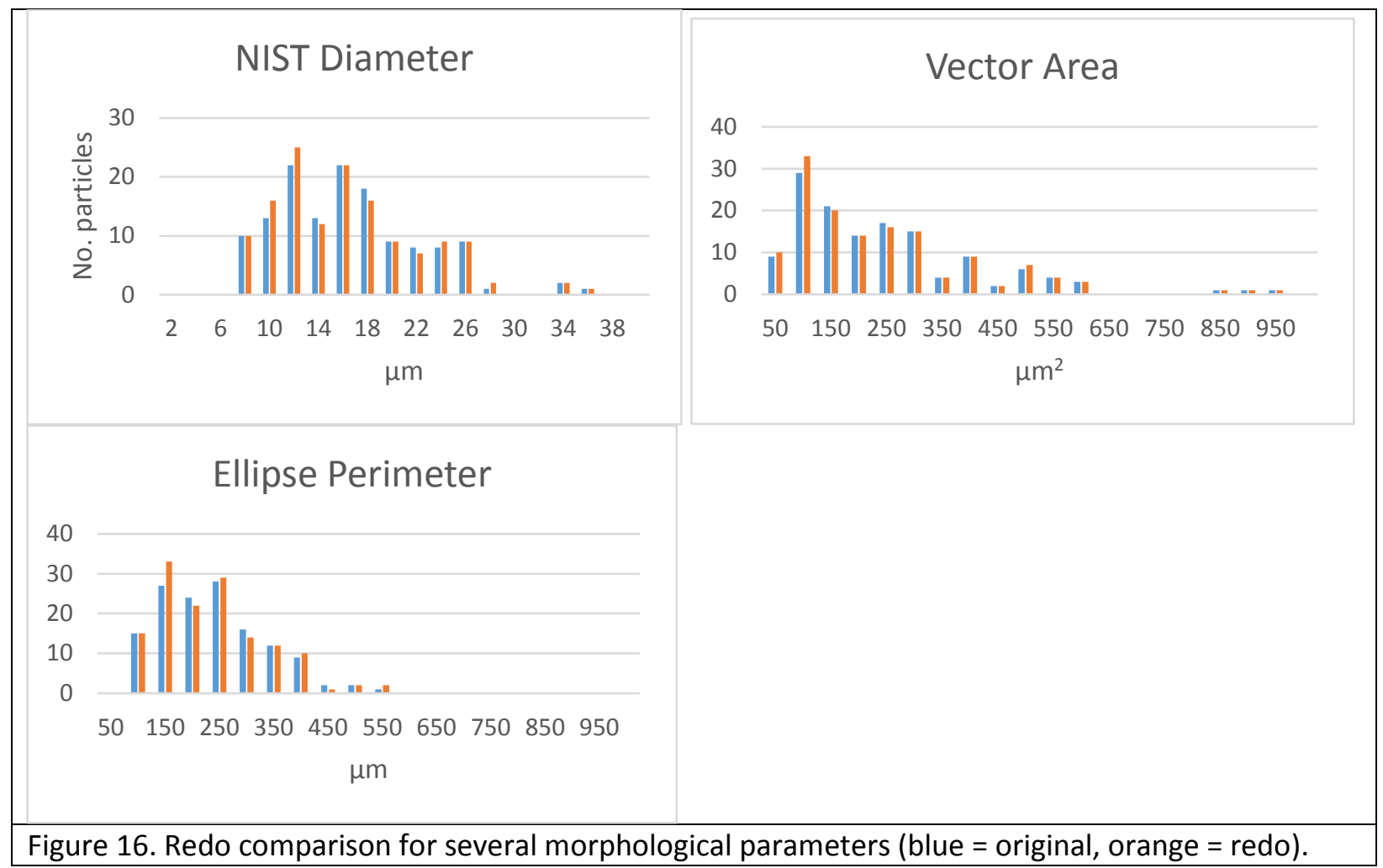

5. SEM imaging mode: There are several imaging modes available on SEMs, two of which are widely used, backscatter electron and secondary electron imaging (BSE, and SE, respectively). The magnification of the BSE and SE modes will only be identical if they are independently calibrated. With the aid of LLNL round robin participants, we compared identical sets of particles imaged using both modes. Figure 9 compares the pixel area morphological parameter from the two sets, and the distributions are almost coincident. A similar comparison plot was made for the vector perimeter (Figure 10). The matching of the vector perimeter is not quite as good, as this morphological parameter depends strongly on the precise positions of edge pixels. However, all the measurements were found to be very similar, and it is clear that as long as there is proper calibration between the two imaging modes, the choice of SEM imaging mode is of secondary importance to morphological quantifications. 

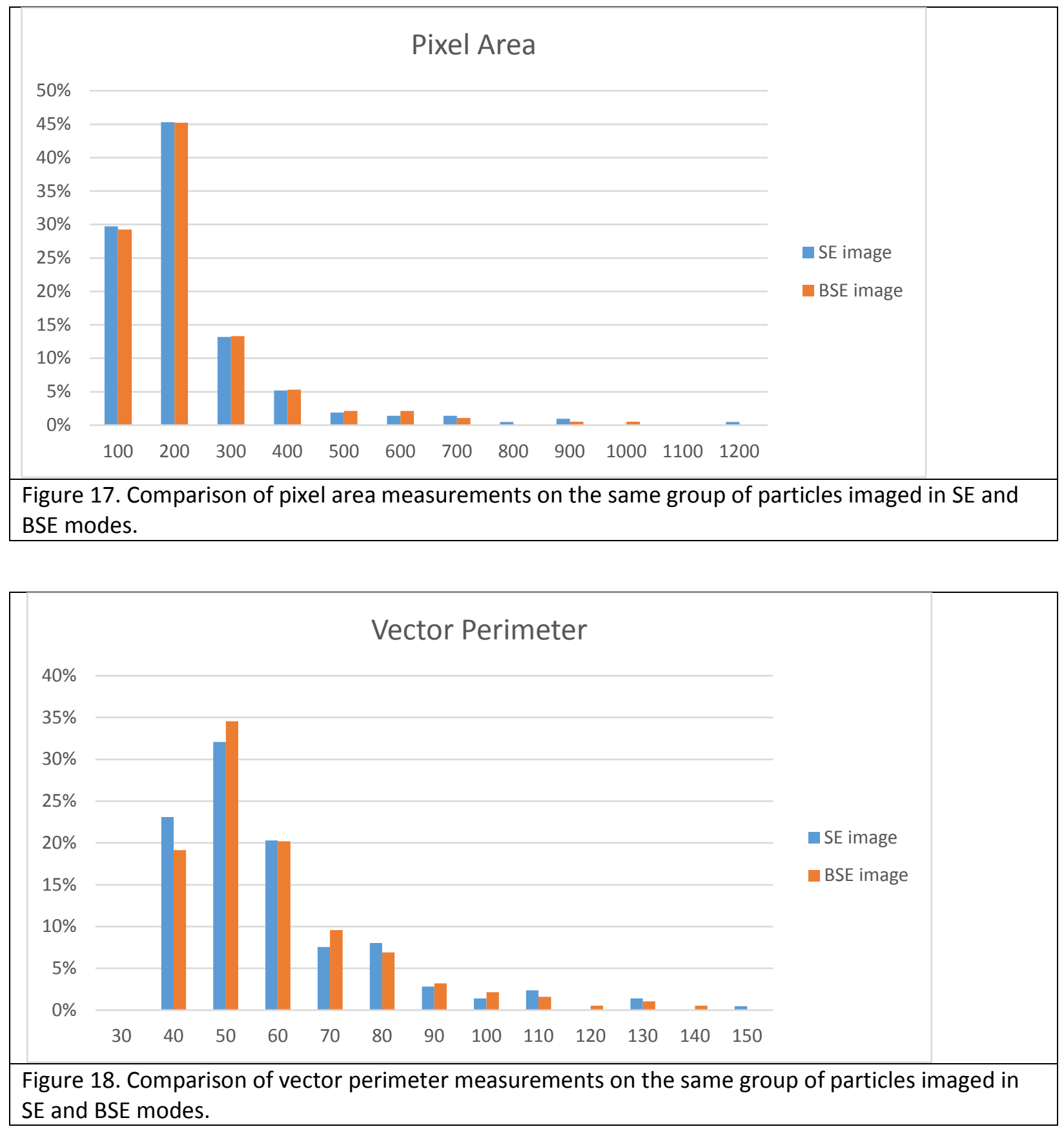

Uncertainty for Level of Rigor 2

A set of 4 SEM specimens was made at LANL for distribution to LLNL, PNNL, and SRNL. As in the Level of Rigor 1 test, the SRM 1984 powder for these was subsampled using a riffler, and a sharp-tipped spatula was used to remove the subsample from its sample bottle. The powder was sprinkled on carbon sticky tape mounted on a standard SEM specimen stub. The 4 specimens were made at the same time, with an attempt to make them as uniform as possible. The same spatula was used throughout, attempting to get 
the same level of loading on each SEM mount. The specimens were sent using Fed Ex to the participating laboratories, and no obvious signs of shipping damage were observed. Using SEMs at their laboratory, each participant made a series of images at suitable magnifications and used the MAMA software to measure $>700$ particles. Level of Rigor 2 is designed to quantify uncertainty introduced by differences in the way analysts make SEM images and calibrate their SEMs, in addition to uncertainties associated with Level of Rigor 1.

Uncertainty for Level of Rigor 2 was evaluated using the same three methodologies used for Level of Rigor 1, namely 1) uncertainty band overlap, 2) histogram analysis, and 3) comparison of cumulative values.

\section{Uncertainty Band Overlap}

Analysis of the data showed that the data from User 6 (U6) was a significant outlier. Figure 11 shows the histogram plot for the NIST diameter data, and the $12 \mu \mathrm{m}$ bin for U6 shows a $10 \%$ higher number of particles than any other analyst. This data set was therefore not included in the analysis, leaving four data sets for developing the uncertainty.

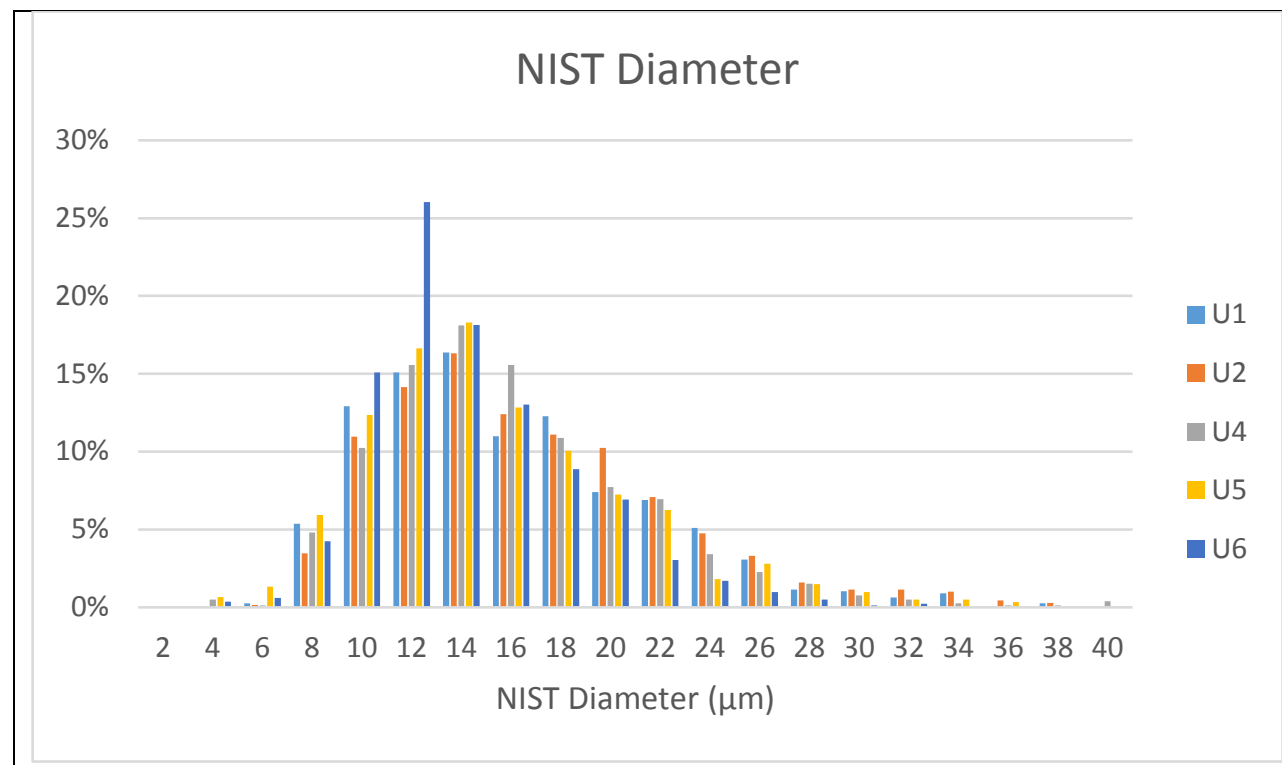

Figure 19. Histogram plot of the NIST diameter data for each analyst.

The confidence band analysis looks at the extreme values for each analysts data, extended by the standard $95 \%$ uncertainty of the value. The results are summarized in Table 2, for each parameter measured by MAMA. Comparing to the Level of Rigor 1 results, we see that the uncertainty is approximately doubled at Level of Rigor 2 . The uncertainties as a percent of the total mean value of 
each parameter is given in the last row of Table 2 . Note that the \pm symbol is meant to indicate that the uncertainty interval extends above and below the mean value.

\begin{tabular}{|c|c|c|c|c|c|c|}
\hline & $\begin{array}{c}\text { Vector } \\
\text { area }\left(\mu \mathrm{m}^{2}\right)\end{array}$ & $\begin{array}{l}\text { Convex hull } \\
\text { area }\left(\mu \mathrm{m}^{2}\right)\end{array}$ & $\begin{array}{c}\text { Pixel area } \\
\quad\left(\mu \mathrm{m}^{2}\right)\end{array}$ & $\begin{array}{c}\text { Vector } \\
\text { perimeter } \\
(\mu \mathrm{m})\end{array}$ & $\begin{array}{c}\text { Convex hull } \\
\text { perimeter } \\
(\mu \mathrm{m})\end{array}$ & $\begin{array}{c}\text { Ellipse } \\
\text { perimeter } \\
(\mu \mathrm{m})\end{array}$ \\
\hline U1 high & 226.07 & 237.78 & 226.06 & 57.96 & 54.12 & 52.64 \\
\hline U1 low & 200.55 & 211.10 & 200.54 & 54.95 & 51.33 & 49.91 \\
\hline U2 high & 247.50 & 263.99 & 248.12 & 63.10 & 57.02 & 55.15 \\
\hline U2 low & 217.56 & 232.49 & 218.15 & 59.68 & 53.93 & 52.12 \\
\hline U4 high & 215.52 & 228.90 & 215.50 & 58.67 & 53.57 & 51.79 \\
\hline U4 low & 193.04 & 205.30 & 193.03 & 55.78 & 50.98 & 49.27 \\
\hline U5 high & 201.55 & 214.61 & 201.53 & 57.03 & 51.64 & 49.88 \\
\hline U5 low & 177.33 & 189.34 & 177.31 & 53.78 & 48.67 & 46.97 \\
\hline $\begin{array}{c}\text { Uncertainty } \\
\text { interval }\end{array}$ & \pm 35.1 & \pm 37.3 & \pm 35.4 & \pm 4.7 & \pm 4.2 & \pm 4.1 \\
\hline $\pm \%$ of mean & $17 \%$ & $17 \%$ & $17 \%$ & $8 \%$ & $8 \%$ & $8 \%$ \\
\hline
\end{tabular}

\begin{tabular}{|c|c|c|c|c|c|}
\hline $\begin{array}{l}\text { Table 2 } \\
\text { cont'd }\end{array}$ & ECD $(\mu \mathrm{m})$ & $\begin{array}{c}\text { Major ellipse } \\
(\mu \mathrm{m})\end{array}$ & $\begin{array}{c}\text { Minor ellipse } \\
(\mu \mathrm{m})\end{array}$ & $\begin{array}{c}\text { Ellipse aspect } \\
\text { ratio }\end{array}$ & $\begin{array}{c}\text { Diameter } \\
\text { aspect ratio }\end{array}$ \\
\hline U1 high & 15.80 & 19.51 & 13.69 & 1.50 & 1.44 \\
\hline U1 low & 14.97 & 18.47 & 12.94 & 1.45 & 1.39 \\
\hline U2 high & 16.53 & 20.39 & 14.41 & 1.48 & 1.42 \\
\hline U2 low & 15.61 & 19.24 & 13.57 & 1.43 & 1.37 \\
\hline U4 high & 15.55 & 19.19 & 13.48 & 1.49 & 1.44 \\
\hline U4 low & 14.79 & 18.23 & 12.78 & 1.44 & 1.39 \\
\hline U5 high & 14.96 & 18.50 & 12.97 & 1.49 & 1.44 \\
\hline U5 low & 14.08 & 17.38 & 12.19 & 1.43 & 1.39 \\
\hline $\begin{array}{c}\text { Uncertainty } \\
\text { interval }\end{array}$ & \pm 1.23 & \pm 1.51 & \pm 1.12 & \pm 0.04 & \pm 0.03 \\
\hline \# \% of mean & $8 \%$ & $8 \%$ & $9 \%$ & $3 \%$ & $3 \%$ \\
\hline
\end{tabular}

\begin{tabular}{|c|c|c|c|c|}
\hline $\begin{array}{l}\text { Table } 2 \\
\text { cont'd }\end{array}$ & Circularity & $\begin{array}{l}\text { Perimeter } \\
\text { convexity }\end{array}$ & Area convexity & NIST diameter \\
\hline U1 high & 0.73 & 0.93 & 0.95 & 15.61 \\
\hline U1 low & 0.72 & 0.93 & 0.94 & 14.80 \\
\hline U2 high & 0.67 & 0.90 & 0.93 & 16.39 \\
\hline U2 low & 0.66 & 0.90 & 0.92 & 15.48 \\
\hline U4 high & 0.70 & 0.92 & 0.94 & 15.37 \\
\hline U4 low & 0.69 & 0.91 & 0.93 & 14.61 \\
\hline U5 high & 0.68 & 0.90 & 0.93 & 14.80 \\
\hline
\end{tabular}




\begin{tabular}{|c|c|c|c|c|}
\hline U5 low & 0.66 & 0.90 & 0.92 & 13.93 \\
\hline $\begin{array}{c}\text { Uncertainty } \\
\text { interval }\end{array}$ & \pm 0.04 & \pm 0.02 & \pm 0.01 & \pm 1.23 \\
\hline $\pm \%$ of mean & $6 \%$ & $2 \%$ & $2 \%$ & $8 \%$ \\
\hline
\end{tabular}

\section{$\underline{\text { Histogram Analysis }}$}

Eliminating the data from U6 results in a significantly smoother distribution for each measured parameter, as seen in Figure 12. The yellow error bars mark \pm 1 standard deviation for each bin in the histogram. As would be expected, the error within each bin is larger than for the Level of Rigor 1 results. The errors for the central bins of the distribution are tabulated in Table 3. The variation within the bins is small enough that we can reliably say that analysts U1, U2, U4, and U5 measured distributions with equivalent shape.

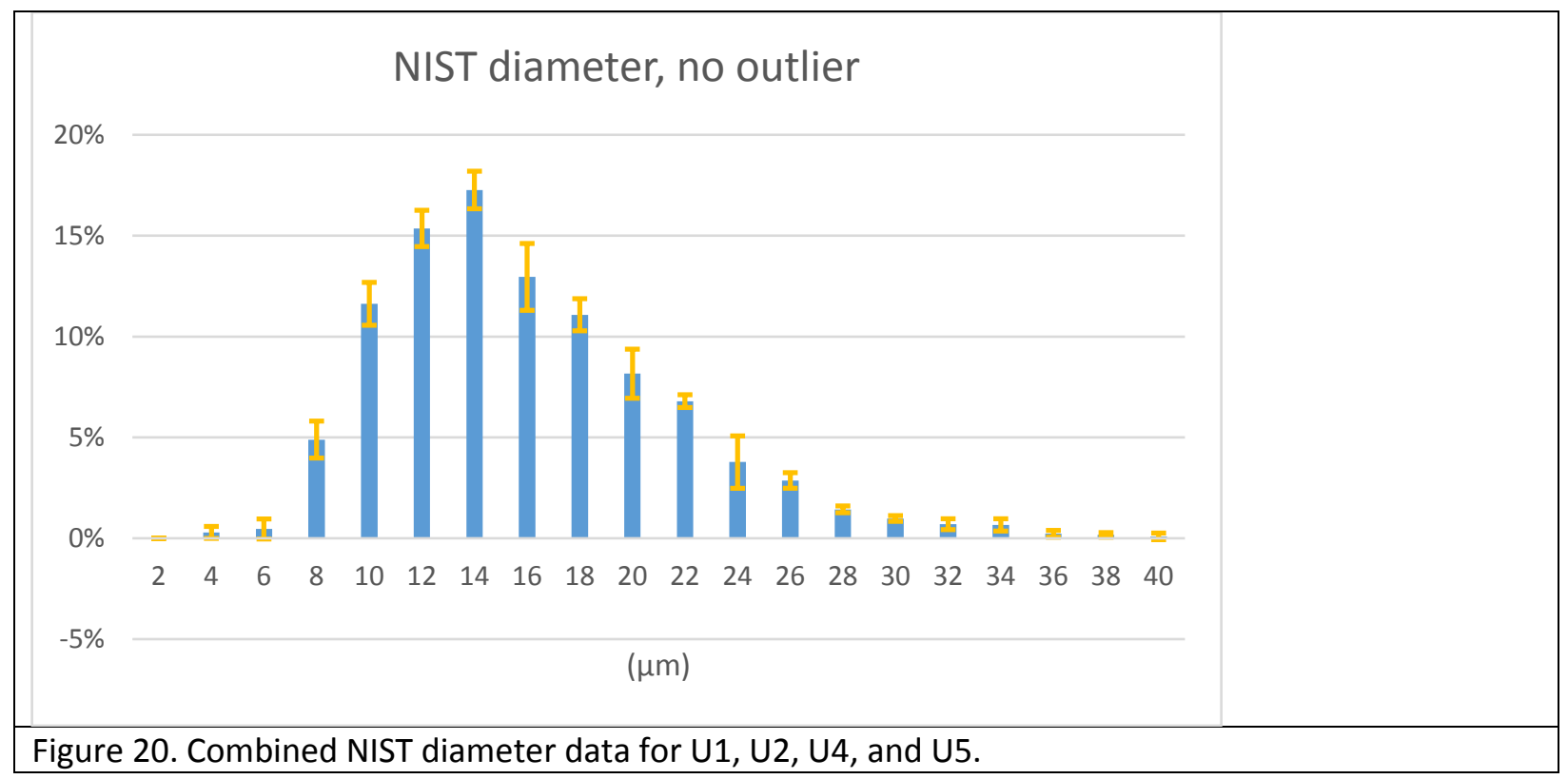

\begin{tabular}{|c|c|}
\hline \multicolumn{2}{|c|}{ Table 10. Histogram analysis for the 4 central bins of the NIST diameter data. } \\
\hline $\operatorname{Bin}(\mu \mathrm{m})$ & $\pm 1-\sigma$ variation within bin \\
\hline $8-10$ & $9 \%$ \\
\hline $10-12$ & $6 \%$ \\
\hline $12-14$ & $5 \%$ \\
\hline $14-16$ & $13 \%$ \\
\hline
\end{tabular}

\section{Cumulative plot analysis}


Cumulative plots for NIST diameter measurements are shown in Figure 13, with and without the inclusion of the outlier data. Again, we see a significant difference in the U6 data, and it will be eliminated from the analysis. Using the remaining data, the spread of the data at $10 \%, 25 \%, 50 \%, 75 \%$, and $90 \%$ cumulative fractions was quantified. The cumulative plots were interpolated to determine the maximum and minimum NIST diameter values at each of the five cumulative fractions, and the difference between these is the error for that specific cumulative fraction (marked with the ellipse in Figure 14). Table 4 shows the results for the NIST diameter data. Again, the uncertainties are significantly higher (roughly $2 x$ higher) than the Level of Rigor 1 results.

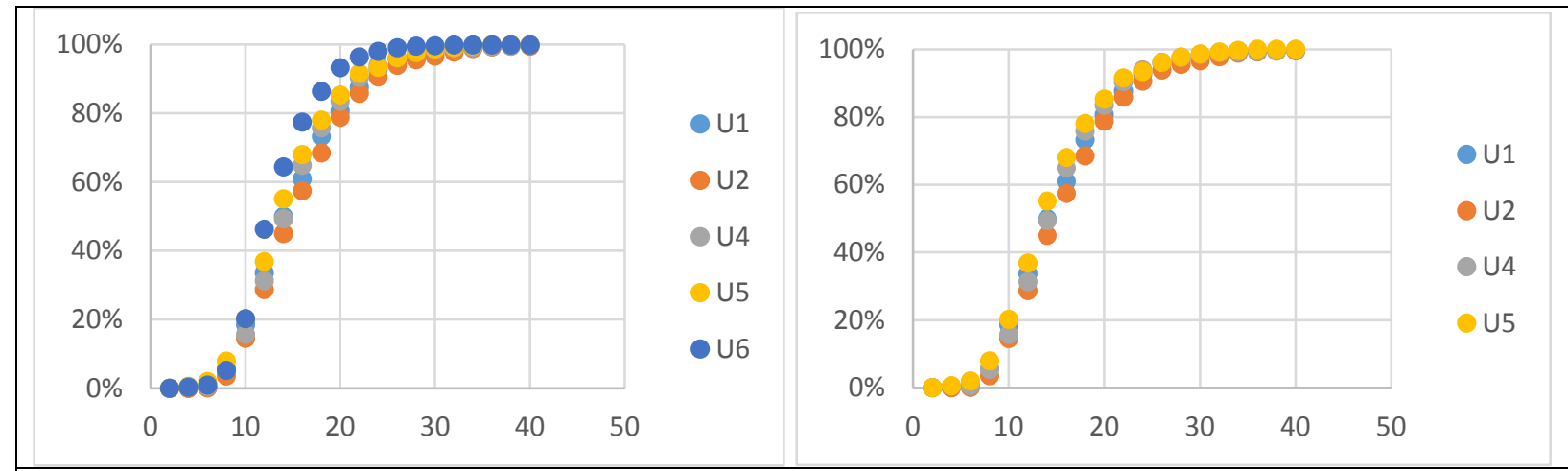

Figure 21. Cumulative frequency plots for NIST diameter, with (left) and without (right) the inclusion of U6 outlier data.

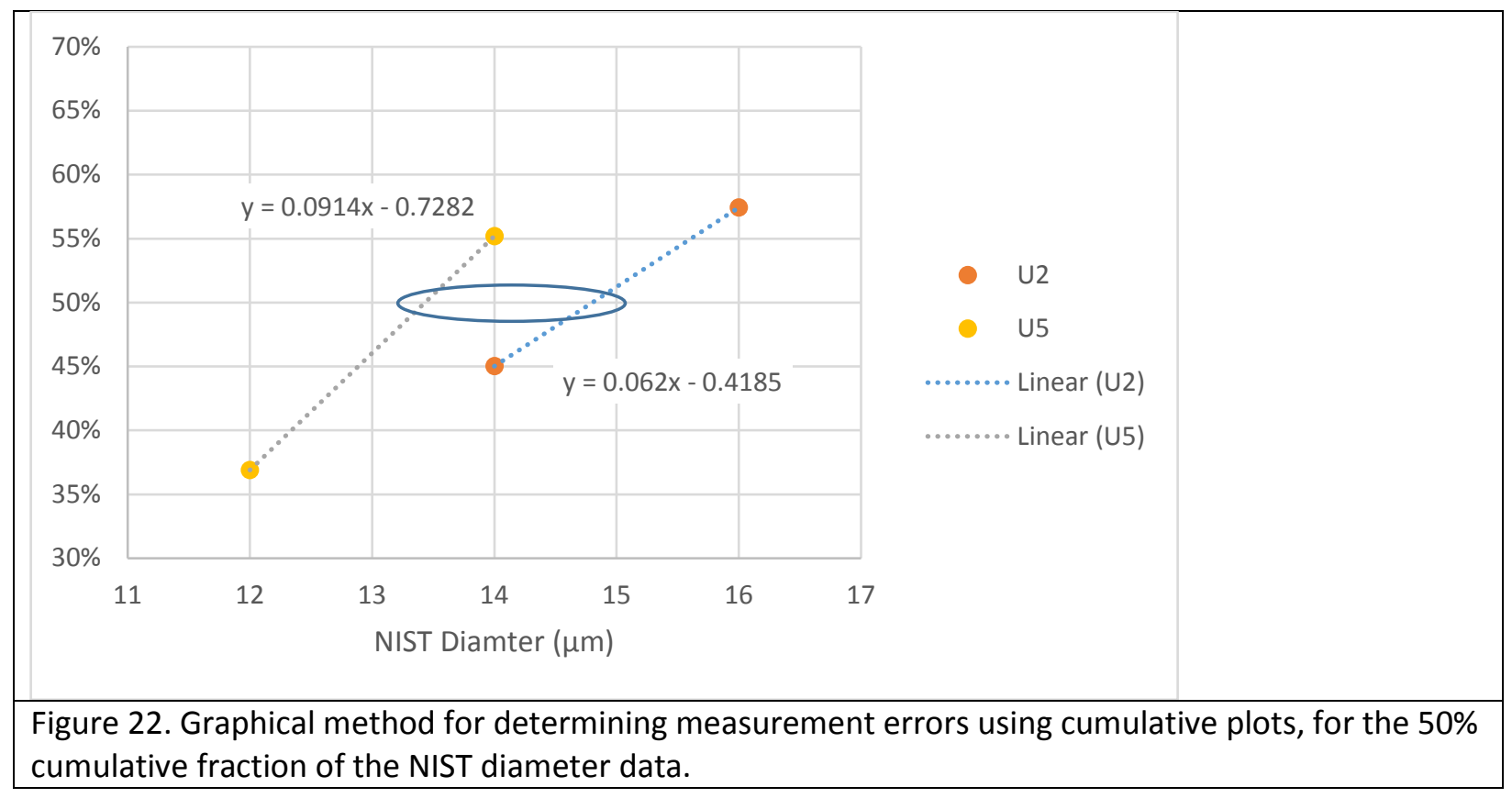

\begin{tabular}{|l|c|c|c|c|}
\hline \multicolumn{6}{|l|}{ Table 11. NIST diameter errors at specific cumulative fractions. } \\
\hline & Minimum value $(\mu \mathrm{m})$ & Maximum value $(\mu \mathrm{m})$ & $\begin{array}{c}\text { Uncertainty } \\
\text { interval }(\mu \mathrm{m})\end{array}$ & $\begin{array}{c} \pm \% \text { around } \\
\text { mean }\end{array}$ \\
\hline
\end{tabular}




\begin{tabular}{|c|c|c|c|c|}
\hline $10 \%$ & 8.3 & 9.2 & 0.8 & $4.6 \%$ \\
\hline $25 \%$ & 10.5 & 11.5 & 1.0 & $4.5 \%$ \\
\hline $50 \%$ (median) & 13.4 & 14.8 & 1.4 & $5.0 \%$ \\
\hline $75 \%$ & 17.4 & 19.3 & 1.9 & $5.2 \%$ \\
\hline $90 \%$ & 21.5 & 23.7 & 2.3 & $5.1 \%$ \\
\hline
\end{tabular}

\section{Level of Rigor 2 conclusions}

A significantly higher uncertainty in MAMA-determined morphological data was measured at this level of rigor. The uncertainty was approximately $2 \mathrm{X}$ higher overall, and reflects the increased uncertainty due to differences in the particle mounting process as well as differences between analyst choices of which portion of the SEM stub to take images from.

Level of Rigor 3

At the highest level of rigor, each participating laboratory made their own SEM mounts, then made their own SEM images, and performed their own MAMA analysis. Uncertainties for Level of Rigor 3 represent the uncertainty we would expect for a real analysis of unknown particulates.

A total of 8 data sets were created and analyzed. Each laboratory riffled their own bottle of SRM 1984 to produce SEM mounts, with one exception. The exception laboratory used the "cone and quarter" technique, in the absence of an available riffler. There was a clear data outlier (possibly due to the use of cone and quartering technique, although one cone and quartered data set looks normal), best seen in the cumulative plot shown in Figure 15. The U5_1 data set contains an anomalously high number of large particles and has a different distribution shape. U5_1 will not be included in the following analysis. Uncertainty for Level of Rigor 3 was evaluated using the same three methodologies used for Level of Rigor 1, namely 1) uncertainty band overlap, 2) histogram analysis, and 3) comparison of cumulative values.

\section{Uncertainty band overlap}

The 95\% uncertainty bands for each analyst's data set were calculated and the maximum spread for each measured morphological parameter was determined. The results are tabulated in Table 5, and comparison to Table 2 shows that the overall 95\% confidence band for all the data at Level of Rigor 3 is very similar to Level of Rigor 2. The main difference between the Level of Rigor 2 and LoR 3 is the addition of riffling and SEM stub mounting steps, so this result shows that the round robin group is fairly uniform in the way that they prepare SEM specimens. Note that the \pm symbol is meant to indicate that the uncertainty interval extends above and below the mean value. 


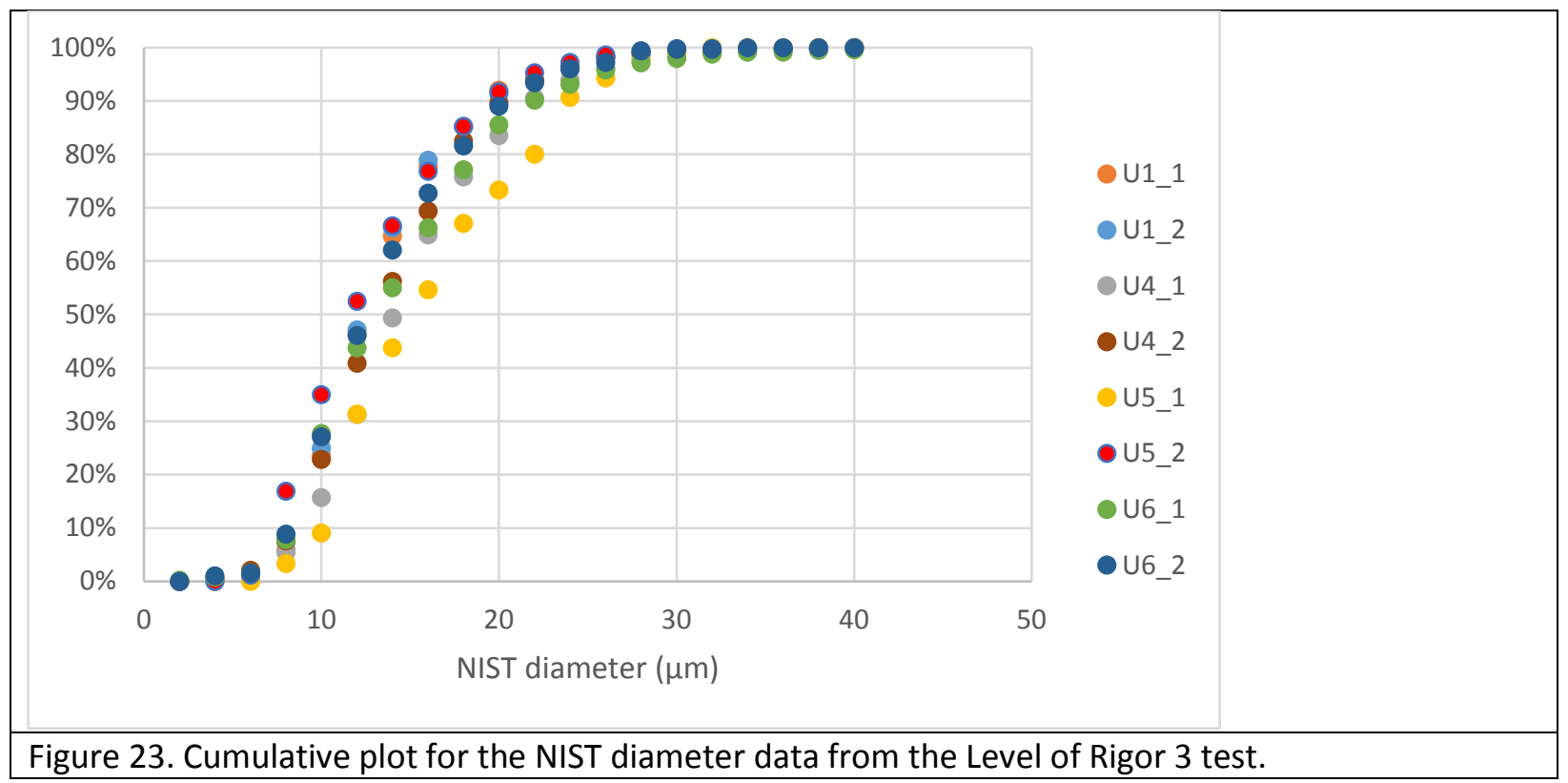

\begin{tabular}{|c|c|c|c|c|c|c|}
\hline & $\begin{array}{c}\text { Vector } \\
\text { area }\left(\mu m^{2}\right)\end{array}$ & $\begin{array}{l}\text { Convex hull } \\
\text { area }\left(\mu m^{2}\right)\end{array}$ & $\begin{array}{c}\text { Pixel area } \\
\left(\mu m^{2}\right)\end{array}$ & $\begin{array}{c}\text { Vector } \\
\text { perimeter } \\
(\mu \mathrm{m})\end{array}$ & $\begin{array}{c}\text { Convex } \\
\text { hull perim } \\
(\mu \mathrm{m})\end{array}$ & $\begin{array}{c}\text { Ellipse } \\
\text { perimeter } \\
(\mu \mathrm{m})\end{array}$ \\
\hline U1_1 max & 169.3 & 182.4 & 169.3 & 52.9 & 48.1 & 46.6 \\
\hline minimum & 147.6 & 159.1 & 147.6 & 49.7 & 45.3 & 43.8 \\
\hline U1_2 max & 168.8 & 179.8 & 168.9 & 51.3 & 47.6 & 46.2 \\
\hline minimum & 147.8 & 157.4 & 147.9 & 48.3 & 44.9 & 43.5 \\
\hline U4_1 max & 215.5 & 228.9 & 215.5 & 58.7 & 53.6 & 51.8 \\
\hline minimum & 193.0 & 205.3 & 193.0 & 55.8 & 51.0 & 49.3 \\
\hline U4_2 max & 183.4 & 195.2 & 183.4 & 54.7 & 49.6 & 47.9 \\
\hline minimum & 164.7 & 175.7 & 164.7 & 52.0 & 47.2 & 45.6 \\
\hline U5_2 max & 164.0 & 175.8 & 172.4 & 51.4 & 46.2 & 44.9 \\
\hline minimum & 138.6 & 148.7 & 137.0 & 48.0 & 43.3 & 41.7 \\
\hline U6_1 max & 208.7 & 223.5 & 208.8 & 57.5 & 51.7 & 50.0 \\
\hline minimum & 176.4 & 188.8 & 176.5 & 53.4 & 48.1 & 46.4 \\
\hline U6_2 max & 173.6 & 186.9 & 175.0 & 54.0 & 48.3 & 46.9 \\
\hline minimum & 154.1 & 166.3 & 155.6 & 51.0 & 45.6 & 44.4 \\
\hline Overall max & 215.5 & 228.9 & 215.5 & 58.7 & 53.6 & 51.8 \\
\hline Overall min & 138.6 & 148.7 & 137.0 & 48.0 & 43.3 & 41.7 \\
\hline $\begin{array}{c}\text { uncertainty } \\
\text { interval }\end{array}$ & \pm 38.5 & \pm 40.1 & \pm 39.3 & \pm 5.3 & \pm 5.2 & \pm 5.0 \\
\hline $\pm \%$ of mean & $22 \%$ & $21 \%$ & $22 \%$ & $10 \%$ & $11 \%$ & $11 \%$ \\
\hline
\end{tabular}

\begin{tabular}{|c|c|c|c|c|c|}
\hline Table 5 cont'd & $E C D(\mu \mathrm{m})$ & $\begin{array}{c}\text { Major ellipse } \\
(\mu \mathrm{m})\end{array}$ & $\begin{array}{c}\text { Minor ellipse } \\
(\mu \mathrm{m})\end{array}$ & $\begin{array}{c}\text { Ellipse aspect } \\
\text { ratio }\end{array}$ & $\begin{array}{c}\text { Diameter } \\
\text { aspect ratio }\end{array}$ \\
\hline
\end{tabular}




\begin{tabular}{|c|c|c|c|c|c|}
\hline U1_1 max & 13.8 & 17.4 & 11.9 & 1.52 & 1.46 \\
\hline minimum & 13.0 & 16.3 & 11.2 & 1.45 & 1.40 \\
\hline U1_2 max & 13.8 & 17.3 & 11.8 & 1.52 & 1.46 \\
\hline minimum & 13.0 & 16.2 & 11.1 & 1.46 & 1.41 \\
\hline U4_1 max & 15.6 & 19.2 & 13.5 & 1.49 & 1.44 \\
\hline minimum & 14.8 & 18.2 & 12.8 & 1.44 & 1.39 \\
\hline U4_2 max & 14.4 & 17.8 & 12.4 & 1.50 & 1.44 \\
\hline minimum & 13.7 & 16.9 & 11.8 & 1.45 & 1.40 \\
\hline U5_2 max & 13.3 & 16.7 & 11.6 & 1.52 & 1.46 \\
\hline minimum & 12.5 & 15.5 & 10.7 & 1.46 & 1.40 \\
\hline U6_1 max & 15.0 & 18.6 & 13.0 & 1.51 & 1.46 \\
\hline minimum & 13.9 & 17.2 & 12.0 & 1.45 & 1.40 \\
\hline U6_2 max & 13.9 & 17.6 & 12.0 & 1.53 & 1.47 \\
\hline minimum & 13.1 & 16.6 & 11.3 & 1.47 & 1.42 \\
\hline Overall max & 15.6 & 19.2 & 13.5 & 1.53 & 1.47 \\
\hline Overall min & 12.5 & 15.5 & 10.7 & 1.44 & 1.39 \\
\hline $\begin{array}{c}\text { uncertainty } \\
\text { interval }\end{array}$ & \pm 1.55 & \pm 1.84 & \pm 1.38 & \pm 0.05 & \pm 0.04 \\
\hline $\pm \%$ of mean & $11 \%$ & $11 \%$ & $11 \%$ & $3 \%$ & $3 \%$ \\
\hline
\end{tabular}

\begin{tabular}{|c|c|c|c|c|}
\hline Table 5 cont'd & Circularity & Perim convexity & Area convexity & NIST diam ( $\mu$ m) \\
\hline U1_1 max & 0.68 & 0.91 & 0.93 & 13.7 \\
\hline minimum & 0.67 & 0.91 & 0.92 & 12.9 \\
\hline U1_2 max & 0.72 & 0.93 & 0.94 & 13.6 \\
\hline minimum & 0.71 & 0.93 & 0.93 & 12.9 \\
\hline U4_1 max & 0.70 & 0.92 & 0.94 & 15.4 \\
\hline minimum & 0.69 & 0.91 & 0.93 & 14.6 \\
\hline U4_2 max & 0.68 & 0.91 & 0.93 & 14.2 \\
\hline minimum & 0.67 & 0.91 & 0.93 & 13.5 \\
\hline U5_2 max & 0.67 & 0.90 & 0.93 & 13.3 \\
\hline minimum & 0.65 & 0.90 & 0.92 & 12.3 \\
\hline U6_1 max & 0.68 & 0.90 & 0.93 & 14.8 \\
\hline minimum & 0.66 & 0.90 & 0.92 & 13.8 \\
\hline U6_2 max & 0.65 & 0.90 & 0.92 & 13.8 \\
\hline minimum & 0.64 & 0.89 & 0.91 & 13.1 \\
\hline Overall max & 0.72 & 0.93 & 0.94 & 15.4 \\
\hline Overall min & 0.64 & 0.89 & 0.91 & 12.3 \\
\hline uncertainty & & & & \pm 1.5 \\
\hline interval & \pm 0.04 & \pm 0.02 & $2 \%$ & $11 \%$ \\
\hline $\pm \%$ of mean & $6 \%$ & $2 \%$ & & \\
\hline
\end{tabular}

$\underline{\text { Histogram analysis }}$ 
The average histogram for NIST diameter data from the Level of Rigor 3 datasets is shown in Figure 16. This histogram is the average of all the data except for the U5_1 outlier. The error bars in Figure 16 mark \pm 1 standard deviation of the data within their associated bin. An analogous histogram for the pixel area data is shown in Figure 17.

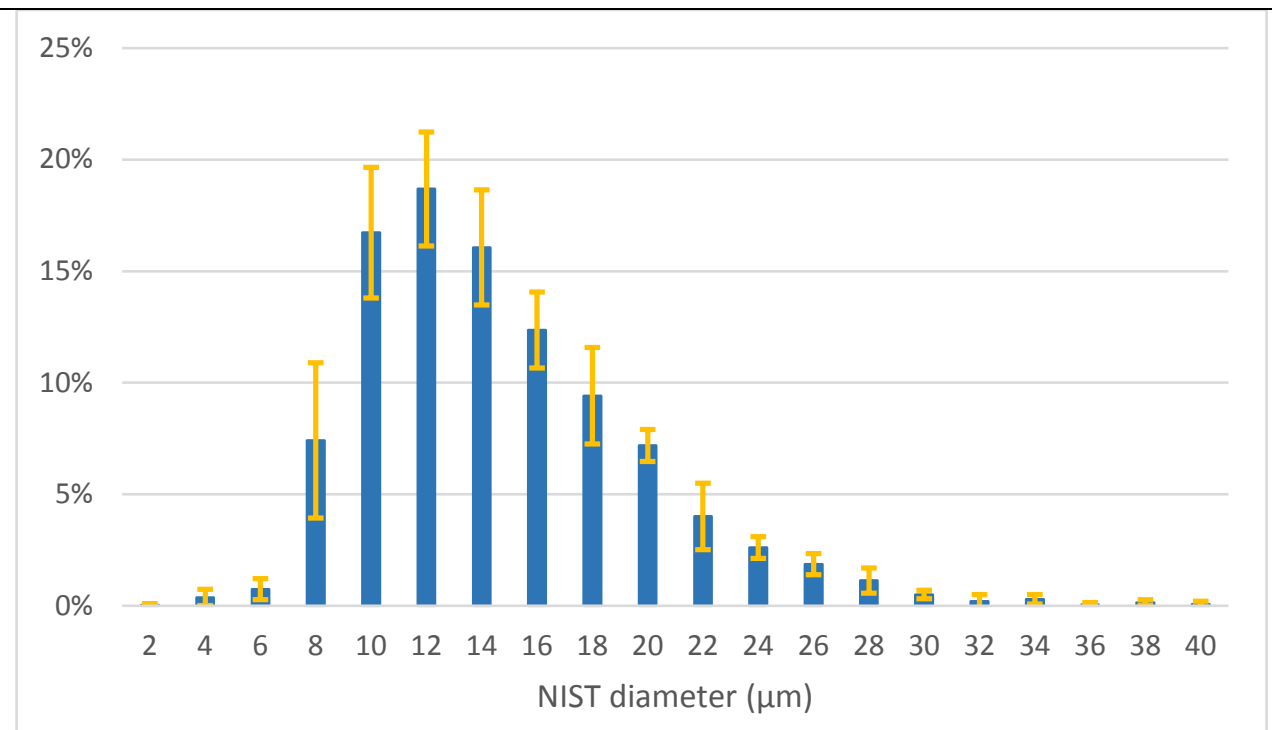

Figure 24. Average histogram for NIST diameter data from the Level of Rigor 3 data set, U5_1 excepted. The error bars mark \pm 1 standard deviation of the data within each bin.

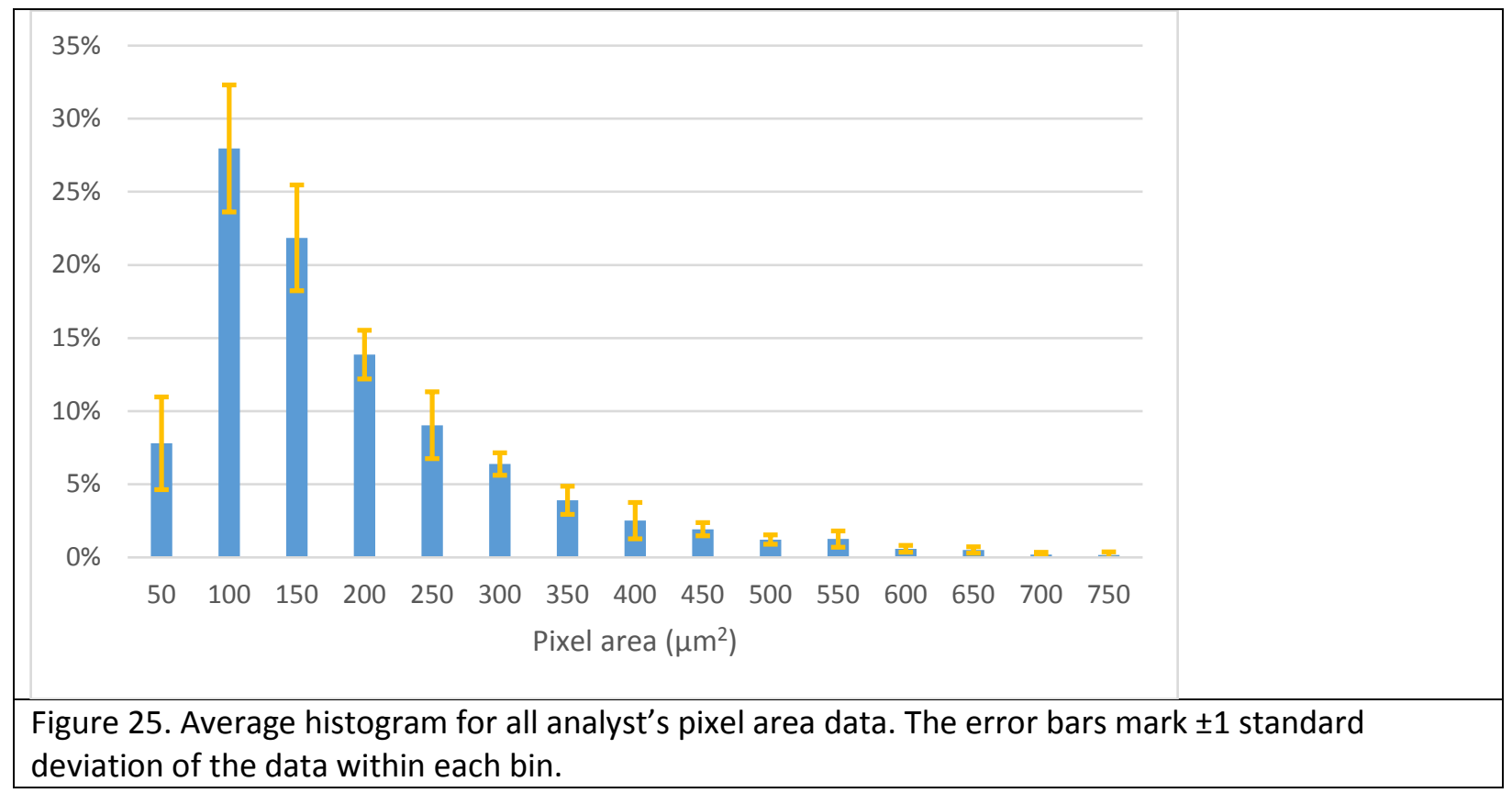


The standard deviation within each of the 4 central bins is tabulated in Table 6 for the NIST diameter histogram (Figure 16). The spread within the bins is larger than that found for Level of Rigor 2, so the process of subdividing and mounting the parent powder for SEM examination introduces more bin-tobin variation in the distribution.

Table 13. Standard deviation of the central bins for the NIST diameter histogram

\begin{tabular}{|c|c|}
\hline Bin range $(\mu \mathrm{m})$ & $\pm 1-\sigma$ within bin \\
\hline $8-10$ & $18 \%$ \\
\hline $10-12$ & $14 \%$ \\
\hline $12-14$ & $16 \%$ \\
\hline $14-16$ & $14 \%$ \\
\hline
\end{tabular}

Cumulative plot analysis

Cumulative plot analysis was performed on the NIST diameter data from each analyst, again with the exception of the U5_1 outlier. The data is shown in Figure 18, overlaid in a single cumulative plot, where it can be seen to be well grouped. The uncertainty was found by measuring the spread of NIST diameter values (see Figure 14 for methodology) at five conventional cumulative fractions, $10 \%, 25 \%, 50 \%, 75 \%$, and $90 \%$. The data is summarized in Table 7. The cumulative uncertainties at Level of Rigor 3 are higher than for Level of Rigor 2, particularly for low cumulative fractions, which is consistent with the larger bin-to-bin variation observed in the histogram analysis.

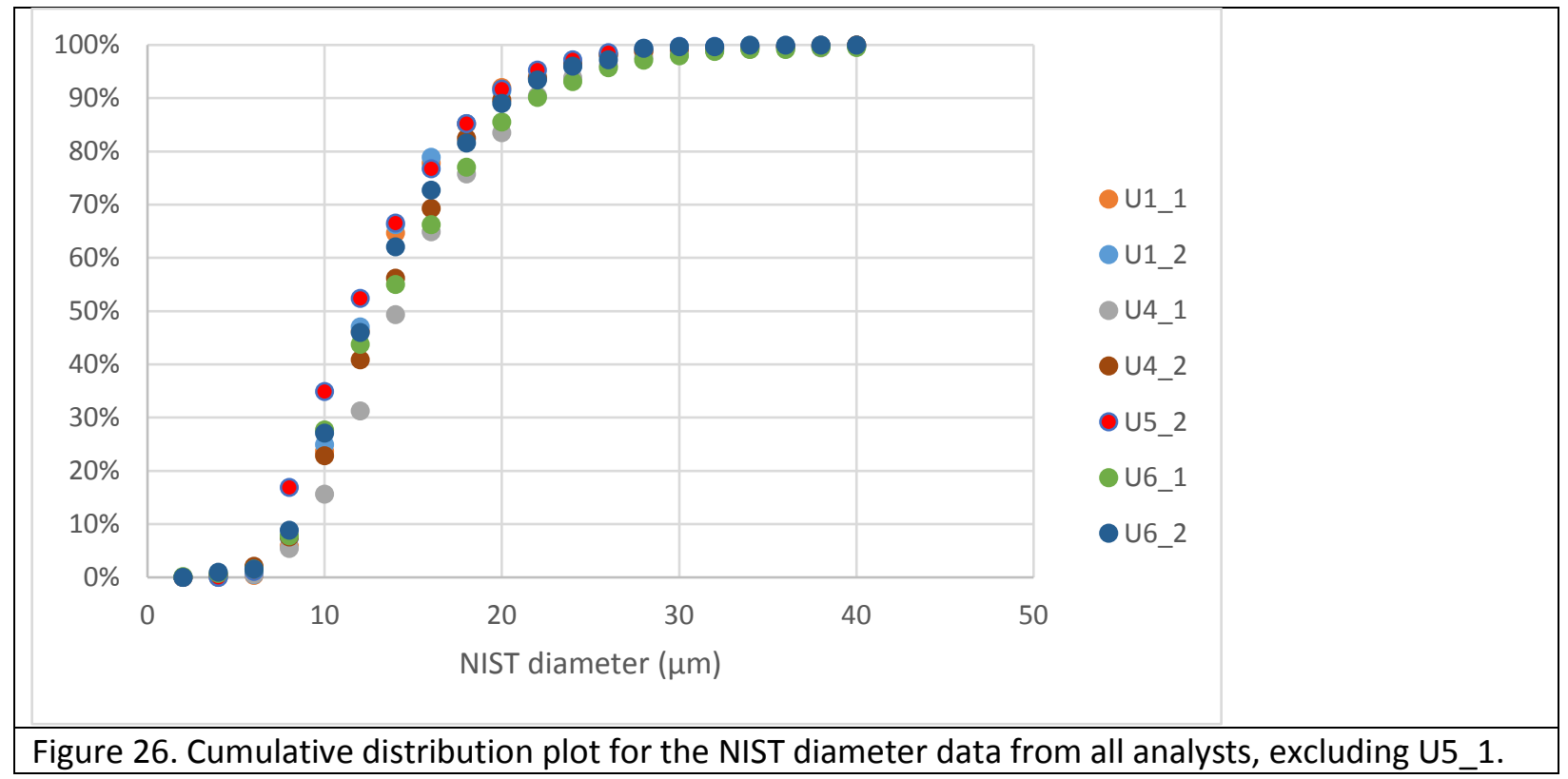




\begin{tabular}{|c|c|c|c|c|}
\hline $\begin{array}{c}\text { Cumulative } \\
\text { fraction }\end{array}$ & $\operatorname{Min}(\mu \mathrm{m})$ & $\operatorname{Max}(\mu \mathrm{m})$ & Uncertainty $(\mu \mathrm{m})$ & $\begin{array}{c} \pm \% \text { around } \\
\text { mean }\end{array}$ \\
\hline $10 \%$ & 7.12 & 8.88 & 1.76 & $11 \%$ \\
\hline $25 \%$ & 8.89 & 11.20 & 2.31 & $11 \%$ \\
\hline $50 \%$ & 11.72 & 14.09 & 2.37 & $9 \%$ \\
\hline $75 \%$ & 15.37 & 17.86 & 2.48 & $7 \%$ \\
\hline $90 \%$ & 19.47 & 21.92 & 2.45 & $6 \%$ \\
\hline
\end{tabular}

\section{$\underline{\text { Acknowledgements }}$}

This work was supported by the TAR program under DNDO (HSHQDC-13-X-B0004). The authors thank Dr. T. Ashenfelter, Program Manager, for his support. 


\section{Appendix A: Final Protocol Document}

$\underline{\text { Introduction }}$

Protocols were developed to minimize variability between different analysts/laboratories for the entire process of sampling from a larger powder set, mounting the sample, imaging the particles, and measuring them using MAMA. Two basic sets of protocols were developed. The first focuses entirely on the use of the MAMA software to quantify particle morphology, while the second addresses procedures to follow when subdividing powder samples, mounting them for SEM analyses, and imaging the mounted particles using SEM.

The protocols have been iterated and improved throughout the program, and the protocols described in this appendix are current best practices. They should not be regarded as fixed, but rather as a set of continuously improving procedures to follow, with improvements incorporated as more analysts use the MAMA software to measure real particle sets.

\section{Protocols for quantitative analysis of SEM images using MAMA}

The protocols described in this section should be followed when making measurements on SEM images of particles (Figure 19 shows a typical SEM image suitable for MAMA analysis). The protocols address only the use of MAMA for quantification. Version 2.0.2 of MAMA should be used, as it is the most current version that has been fully verified and validated.

1. The preferred method for establishing the magnification of SEM images is by developing a calibration curve for the SEM, using a traceable SEM magnification standard (see the next section). In the absence of a calibration curve, step 2 below applies.

2. The magnifications for each image may be determined by using the size bar saved in the image by the SEM software and the RULER

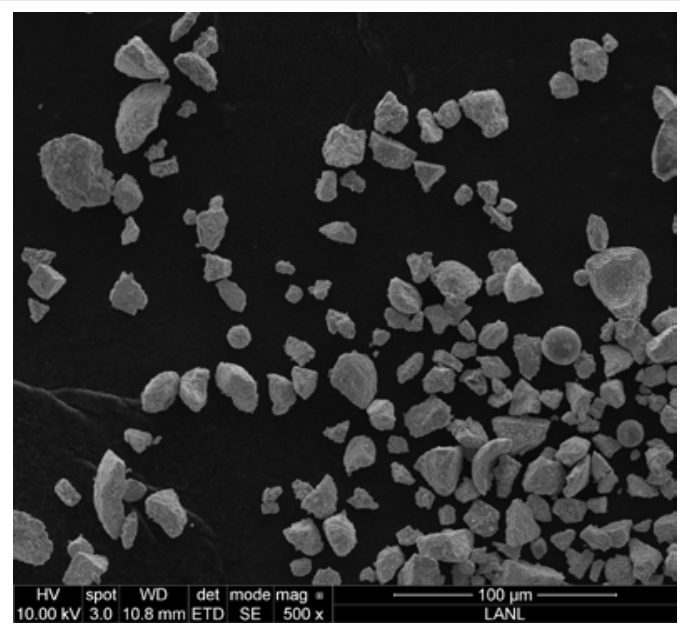

Figure 27. Typical SEM image of SRM 1984 for use in round-robin morphological analysis. function in the MAMA software. The precision of this process can be significantly increased by magnifying the image display in MAMA several steps before using the click-and-drag magnification tool in the RULER function. Users should report whether a calibration curve or the RULER tool was used to determine the magnification of the image. In addition, users should report the magnification value in pixels/ $\mu \mathrm{m}$ for each image (NOTE: this information is stored by MAMA in the metadata file created when the analysis data is exported). 
3. Users should try to measure $>700$ particles. This number gives a good, well-understood statistical sample. However, including more particles will always give a more reliable result.

4. Users should image their particle sets at a variety of magnifications, with the goal of obtaining clear images of particles at all the size scales present in the sample.

5. User should report the imaging mode they used for the SEM images (BSE or SE).

6. Users should set MAMA to calculate all the morphological parameters, with minimum size $=1000$ pixels, and "Correct for vector bias" ON. These settings are under menu item Analysis $\rightarrow$ Options $\rightarrow$ Quantification Options.

7. All particles overlapping or touching any edge of the images must be excluded from the measurements. Note that MAMA has multiple ways to exclude particles (Figure 20).

8. Overlapped particles shall be excluded from the measurements (Figure 21).

a. The foreground particle in an overlap may be included if its boundary is welldefined.

b. If particles are simply touching, they may be separated using the SPLIT SEGMENT tool. Some user judgement will be required to decide when to split particles instead of excluding them. However, error due to a modest overlap

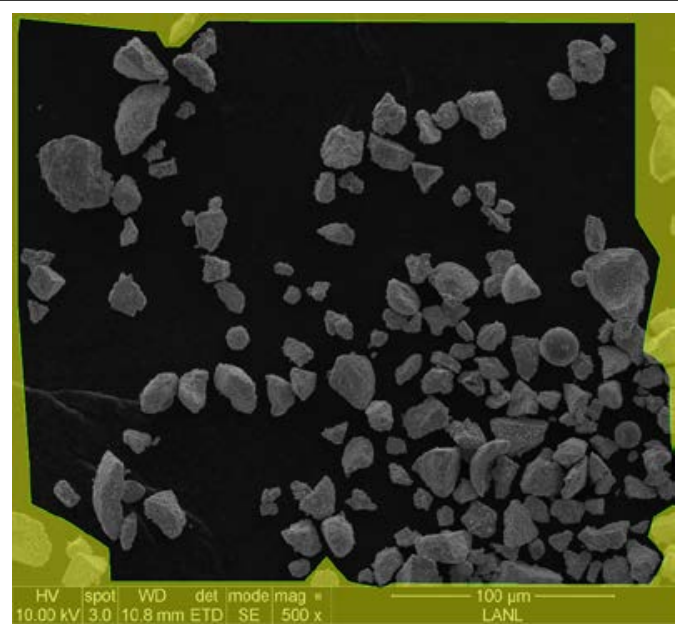

Figure 28. Particles overlapping the image edge are eliminated from the analysis. will be much smaller than the $95 \%$ confidence band associated with the 700 particle population.

9. Analysts should try to measure all particles in a given image (within the restraints of steps 7 and 8). Any analyst choice of particles will bias the data.

10. When segmenting, use the "smoother" filter, as a good compromise segmentation filter. Normally, this is the default mode, but if it is not selected, select it from the pull-down menu that appears when the SEGMENT function is selected. The choice of segmentation filter has a significant quantitative effect on the data, and the choice of filter should always be reported. Note that this information is not currently saved with the MAMA data sets.

11. Prior to finalizing the data set, analysts should review their segmented images for any obvious mis-segmentations. In general, it is better to exclude a questionable particle even though this will make it more difficult to get to the $>700$

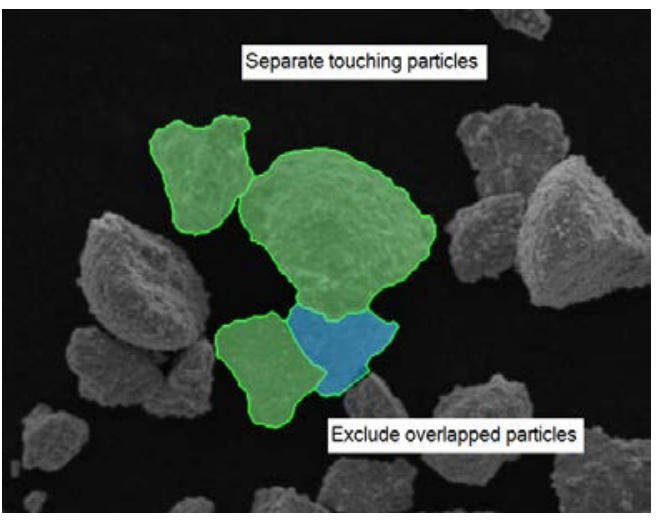

Figure 29. Separate touching particles, and remove overlapped particles from analysis. particle goal. 
a. SUGGESTION: After a performing a segmentation, look at the histogram for pixel area (a function in the QUANTIFY tool set). If there are anomalously small values, go back to the image and find those small segments. If they are not real particles (often they are missegmentations within a larger particle), merge them into their associated particle and rerun the quantification.

12. After quantifying, the data is automatically saved in the MAMA software, along with the image and workflow. It is also useful to save the data as a text file, which will also create a metadata file with the same name. The data is then easily imported into Excel or other data processing software.

Protocols for subdividing, mounting, and imaging powder sets for analyzing particles with MAMA

Protocols described in this section will necessarily depend on a high level of analyst skill. The process of properly mounting specimens and imaging them using SEM is a skilled operation, developed over years of experience. However, following the general steps below will help make analyses more uniform and comparable.

1. It is best to prepare and analyze multiple SEM mounts from a powder set. There will always be some variation between mounts and this will help quantify the variation.

2. Parent powder sampling should be done using a riffler, when the parent set is large enough ( $\mathrm{mL}$ level). Note that riffling must initially be done on the entire parent set to avoid bias. It is best to perform multiple riffling steps and riffle the parent powder set down to a final subset of $\sim 1 \mu \mathrm{L}$.

3. Final sub-sampling for the purposes of SEM mounting should be done using a sharp-tipped spatula. Sharp-tipped spatulas cut from aluminum foil are suitable, as are any fine-tipped, concave, commercially available spatulas.

4. Deliver the powder onto SEM stubs covered with conductive sticky tape. It is preferable to have the powder distributed at a low density to avoid excessive particle overlaps, which complicates the MAMA morphological analysis.

5. Sufficient SEM images should be captured so that $>700$ particles total can be measured by MAMA. Regions for imaging should be chosen as randomly as possible. Over-selection by the SEM operator can bias the quantification of particle morphology.

6. Magnification should be varied such that the micrograph set includes clear images of both the smallest and largest particles in the sample. Ideally, the smallest particles in the highest magnification images should contain $>1000$ pixels.

a. NOTE: At 1000x magnification, a $1 \mu \mathrm{m}$ diameter particle will have roughly 100-200 pixels, well below the target number.

7. SEM operators should calibrate the magnifications of their instruments using a traceable calibration standard, such as the Geller MRS-3 or MRS-4.2 magnification standard. Operators should report the calibration in the form of a calibration curve or table (pixels/ $\mu \mathrm{m}$ for each magnification). Magnification in both $X$ and $Y$ directions shall be reported. This value for pixels $/ \mu \mathrm{m}$ should be manually entered into the MAMA software RULER tool to set the magnification that is used by MAMA. 
a. NOTE: A calibration curve must be developed for each SEM imaging mode. Backscatter and secondary electron imaging will not necessarily have identical calibration curves, and need to be independently calibrated.

8. Magnification calibration of SEMs should be performed at least annually, and following any major instrument repair involving magnetic lens control or beam column modification. Calibrations should be done using a standard specimen height (objective-to-specimen focal plane distance). This height should then be used for imaging the powder set.

9. SEM parameters such as voltage, spot size, and image mode (backscatter vs. secondary electron) shall be left to the discretion of the analyst. It is good practice to report this information, or include it in an image footer. Analysts should use their expertise to capture sharp particle images with good edge contrast. 